\title{
Mechanistic insights into the enhancement or inhibition of phase separation by polyubiquitin chains of different lengths or linkages
}

Thuy P. Dao ${ }^{1, \#, ~ Y i r a n ~ Y a n g ~}{ }^{2, \#}$, Michael S. Cosgrove ${ }^{3}$, Jesse B. Hopkins ${ }^{4}$, Weikang Ma ${ }^{4}$, Carlos A. Castañeda ${ }^{1,5}$

${ }^{1}$ Departments of Biology and Chemistry, Syracuse University, Syracuse, NY 13244, USA

2 Department of Chemistry, Syracuse University, Syracuse University, Syracuse, NY 13244, USA

${ }^{3}$ Department of Biochemistry and Molecular Biology, SUNY Upstate Medical University, Syracuse, NY 13210, USA

${ }^{4}$ The Biophysics Collaborative Access Team (BioCAT), Department of Biological Sciences, Illinois Institute of Technology, Chicago, IL 60439, USA

${ }^{5}$ Interdisciplinary Neuroscience Program, Syracuse University, Syracuse University, Syracuse, NY 13244, USA

\# These authors contributed equally.

* Corresponding Author cacastan@syr.edu 


\section{Summary}

Ubiquitin-binding shuttle UBQLN2 mediates crosstalk between proteasomal degradation and autophagy, likely via interactions with K48- and K63-linked polyubiquitin chains, respectively. UBQLN2 is recruited to stress granules in cells and undergoes liquid-liquid phase separation (LLPS) in vitro. However, interactions with ubiquitin or multivalent K48-linked chains eliminate LLPS. Here, we found that, although some polyubiquitin chain types (K11-Ub4 and K48-Ub4) did generally inhibit UBQLN2 LLPS, others (K63-Ub4, M1-Ub4 and a designed tetrameric ubiquitin construct) significantly enhanced LLPS. Using nuclear magnetic resonance (NMR) spectroscopy and complementary biophysical techniques, we demonstrated that these opposing effects stem from differences in chain conformations, but not in affinities between chains and UBQLN2. Chains with extended conformations and increased accessibility to the ubiquitin binding surface significantly promoted UBQLN2 LLPS by enabling a switch between homotypically to partially heterotypically-driven phase separation. Our study provides mechanistic insights into how the structural and conformational properties of polyubiquitin chains contribute to heterotypic phase separation with ubiquitin-binding shuttles and adaptors.

\section{Highlights}

- Ubiquitin or short polyubiquitin chains bind to phase separation-driving stickers on UBQLN2 and inhibit its phase separation whereas longer chains provide the multivalency needed to enhance UBQLN2 phase separation.

- Compact K11- and K48-linked Ub4 chains destabilized UBQLN2 phase separation, while extended M1- and K63-linked Ub4 promoted UBQLN2 phase separation.

- Chain conformation and accessibility of the Ub interacting surface is a driving factor of UBQLN2/polyUb co-phase separation.

- UBQLN2 condensates assemble during in vitro enzymatic assembly of K63-linked polyUb chains as free ubiquitin is depleted. 


\section{Introduction}

Protein quality control (PQC) mechanisms, which enable cells to combat aberrant proteins, is essential for cellular functions and viability (Dikic, 2017; Vendruscolo, 2012). Several distinct but connected pathways, such as proteasomal degradation, endoplasmic reticulumassociated protein degradation and autophagy exist to ensure proper PQC. In most cases, ubiquitination of substrate proteins is the common signal for these different pathways, as well as numerous other processes, such as DNA damage response, cargo transport and cell cycle control (Komander and Rape, 2012). This wide range of cellular responses to ubiquitination stems from the ability of ubiquitin ( $\mathrm{Ub}$ ) to form polyubiquitin (polyUb) chains with different lengths and linkages. The two most abundant and well-studied polyUb chain types are K48- and K63-linked chains that generally signal for proteasomal degradation and autophagy, respectively. Other chains, such as K11- and M1-linked chains, are involved in degradative and non-proteolytic pathways, respectively (Akutsu et al., 2016). It is hypothesized that polyUb chains elicit different biological signaling outcomes based on differences in their conformational properties and how each chain type interacts with Ub-binding receptors, such as shuttle proteins (Komander and Rape, 2012). Different shuttle proteins can recognize specific chains and transport modified substrates to distinct pathways (Komander and Rape, 2012). Some shuttle proteins, such as autophagy receptor NBR1 and proteasomal shuttle protein hHR23B, are mostly pathway specific. Others, such as UBQLN2 and p62, are involved in multiple pathways (Zientara-Rytter and Subramani, 2019). How shuttle proteins choose a specific pathway upon binding to ubiquitinated substrates, possibly through changes in conformations or oligomeric states, is of great interest (Lu et al., 2017).

PQC impairment due to aging, prolonged stress or mutations can lead to proteincontaining inclusions characteristic of neurodegenerative diseases, such as amyotrophic lateral sclerosis (ALS), Parkinson's and Alzheimer's diseases (Hipp et al., 2014; Labbadia and Morimoto, 2015). These inclusions often comprise Ub, polyUb chains, Ub-binding shuttle proteins and other PQC proteins (Lowe et al., 1988; Manetto et al., 1988; Morimoto et al., 2015; Riley et al., 2010). Many of these proteins are also part of membraneless compartments, such as stress granules and PQC compartments that sequester aberrant proteins in preparation for clearance in healthy eukaryotic cells (Sontag et al., 2017). Dysregulation of membraneless compartments can lead to disease-linked inclusions (Molliex et al., 2015; Nedelsky and Taylor, 2019; Patel et al., 2015; Ryan and Fawzi, 2019). Liquid-liquid phase separation (LLPS) of a few key scaffolding proteins drive the formation of membraneless compartments whereas interacting partners of scaffolding proteins can modulate the properties of these compartments (Guillén- 
Boixet et al., 2020; Sanders et al., 2020; Yang et al., 2020; Yasuda et al., 2020). Therefore, knowledge of how binding partners affect phase-separating scaffolds is essential for understanding the regulation of $\mathrm{PQC}$ mechanisms.

We recently showed that Ub-binding shuttle protein UBQLN2 phase separates under physiological conditions but specific interactions with Ub, K48-linked Ub2 and Ub4 disrupt UBQLN2 LLPS (Dao et al., 2018). Following the polyphasic linkage formalism put forth by Wyman and Gill, UBQLN2 is considered a scaffold that drives condensate formation and Ub/K48 polyUb chains are ligands that can modulate LLPS (Wyman and Gill, 1980). Ligands can either enhance or inhibit LLPS, depending on their multivalency (in this case, the number of Ub units in a chain) and binding preferences for the scaffold in the dense and dilute phases (Ruff et al., 2021a). Unlike UBQLN2, proteasomal shuttle protein hHR23B and autophagy receptor p62 require longer K48- and K63-linked polyUb chains, respectively, to phase separate and carry out their functions (Sun et al., 2018; Yasuda et al., 2020). Interestingly, UBQLN1, which is a close homolog of UBQLN2 and is involved in both autophagy and proteasomal degradation, might bind preferentially to K63- over K48-linked chains (Harman and Monteiro, 2019). Therefore, we hypothesized, and showed in this work, that different polyUb chain types elicit distinct effects on UBQLN2 LLPS. By studying the interactions between UBQLN2 and various naturally occurring and designed polyUb chains, we determined the molecular mechanisms underlying these drastically different effects on UBQLN2 LLPS. Our results offer insights into how polyUb chains of distinct linkages drive ligand-induced phase transitions, and how modulation of UBQLN2 LLPS may steer UBQLN2 involvement into various PQC pathways. 


\section{Results}

PolyUb chains with distinct linkage types and lengths differentially affect UBQLN2 LLPS

To determine if various polyUb chains impact UBQLN2 LLPS behavior differently, we initially focused on enzymatically synthesized K48 and K63 chains of two, three and four Ub units (Fig. S1). These two chains are the most abundant in cells, signal for different cellular events, exhibit distinct solution conformations, and selectively drive LLPS of other Ub-binding shuttle proteins (Castañeda et al., 2016a; Dao and Castañeda, 2020; Sun et al., 2018; Yasuda et al., 2020; Ye et al., 2012).

We first screened for the effects of Ub chains on full-length (FL) UBQLN2 LLPS by observing UBQLN2 droplets with fluorescence microscopy at different Ub:UBQLN2 ratios (Fig. 1A). Consistent with our previous data, increasing amounts of Ub disassembled UBQLN2 droplets (Dao et al., 2018, 2019). Using nuclear magnetic resonance (NMR) spectroscopy, we confirmed that Ub binds only to the Ub-associated (UBA) domain in UBQLN2 with a $K_{d}$ of $3 \mu M$ (similar to what we reported for Ub binding to UBQLN2 450-624 (Dao et al., 2018)); only amide peaks of UBA residues shifted in the presence of Ub (Fig. S2A). As Ub binds to the same UBA sites that are important for UBQLN2 LLPS (Fig. S2B, (Dao et al., 2018)), we classified Ub as a monovalent ligand that interacts with UBA stickers and destabilizes LLPS (Ruff et al., 2021a).

In contrast to Ub, K48- and K63-Ub2, Ub3, and Ub4 chains can be considered di-, triand tetra-valent ligands that bind to UBQLN2. We observed several trends when mixing these multivalent ligands with UBQLN2: 1) Droplets persisted at increasingly higher Ub:UBQLN2 ratios as chain length/multivalency increased, regardless of linkage type. 2) Unlike K48 chains, longer K63 chains enhanced UBQLN2 LLPS at lower Ub:UBQLN2 ratios (larger droplets likely resulted from the sample containing more smaller droplets that fused and settled on the bottom of the coverslip where imaging was done). 3) All chains inhibited LLPS at higher ratios (Fig. 1A).

\section{K63-Ub4 promotes reentrant phase behavior of UBQLN2}

To quantitatively describe the effects of polyUb on UBQLN2 LLPS, we ran turbidity experiments that monitored the change in $A_{600}$ values as a function of temperature at different Ub:UBQLN2 ratios. We previously showed that high and low $A_{600}$ values correlate with droplet formation and clearance, respectively, and that UBQLN2 phase separates with increasing temperature (Dao et al., 2018). Increasing amounts of Ub and K48-Ub2 reduced and eventually eliminated UBQLN2 LLPS (Fig. 1B and (Dao et al., 2018)). The turbidity plots for K63-Ub2 
addition to UBQLN2 were nearly identical to those of K48 Ub2 and Ub, suggesting that lysine linkage does not matter at the Ub2 level.


Figure 1. PolyUb chain length and linkage affects UBQLN2 LLPS. (A) Fluorescence microscopy showing enhancement or inhibition of UBQLN2 droplet formation with increasing amounts of K48- and 
K63-linked chains of different lengths. All solutions contained $50 \mu \mathrm{M}$ full-length UBQLN2 in $20 \mathrm{mM}$ NaPhosphate and $200 \mathrm{mM} \mathrm{NaCl}\left(\mathrm{pH} \mathrm{6.8)}\right.$ at $30^{\circ} \mathrm{C}$. For direct comparison among different chain lengths, [Ub]:[UBQLN2] reflects the ratio between Ub monomers and UBQLN2 molecules. Solution is spiked with Alexa Fluor 647-labeled UBQLN2. The same image is used for the 0:1 condition. Scale bar, $5 \mu \mathrm{m}$. Yellow cartoons on the right illustrate one major solution conformation of each chain. (B) Representative results from spectrophotometric turbidity assay as a function of temperature comparing the effects of varying amounts of K48- and K63-linked chains of different lengths on UBQLN2 LLPS at $30 \mu M$ UBQLN2. (C) Temperature-composition phase diagrams showing changes in cloud point temperatures as a function of Ub:UBQLN2 for different chains and different UBQLN2 concentrations. The black dashed lines denote biologically relevant temperature $37^{\circ} \mathrm{C}$, at which $12.5 \mu \mathrm{M}$ UBQLN2 only undergoes LLPS with K63-Ub4.

Addition of Ub3 and Ub4 chains of different linkages led to distinct turbidity plots (Fig. 1B), consistent with our microscopy data (Fig. 1A). Strikingly, K63-Ub4 visibly promoted and inhibited LLPS, depending on Ub:UBQLN2 ratio, reminiscent of reentrant phase behavior seen for protein-RNA coacervates that form via heterotypic interactions (Alshareedah et al., 2019; Banerjee et al., 2017). Low K63-Ub3/Ub4:UBQLN2 ratios significantly promoted LLPS. As these ratios were further increased, LLPS was inhibited. We hypothesized that K63-Ub4 promotes LLPS by acting as an emergent scaffold onto which multiple UBQLN2 molecules bind. These heterotypic interactions provide additional multivalency to further promote LLPS. However, as Ub4 concentrations increase, not enough UBQLN2 molecules exist to bind Ub4 simultaneously, hence diluting out the LLPS-driving UBQLN2-UBQLN2 interactions and leading to inhibition of LLPS (Banerjee et al., 2017; Choi et al., 2019; Dao and Castañeda, 2020; Ruff et al., 2021b). Conversely, like Ub and K48-Ub2, K48-Ub3 also inhibited LLPS at all concentrations. Low K48Ub4:UBQLN2 ratios slightly enhanced LLPS whereas high ratios inhibited LLPS.

To quantitatively compare the effects of Ub and polyUb on UBQLN2 LLPS, we obtained temperature-composition phase diagrams for different Ub:UBQLN2 ratios at multiple UBQLN2 concentrations (Fig. 1C). We determined $\mathrm{T}_{\mathrm{cp}}(\mathrm{infl})$, the cloud point temperature at the inflection point of the transition, as done previously (Yang et al., 2019). We used $T_{c p}$ at different Ub:UBQLN2 ratios to map out the coexistence curve, above which the protein solution is phase separated. For Ub and Ub2, we observed roughly linear increase in $T_{c p}$ as Ub:UBQLN2 ratio increased, consistent with Ub and Ub2 being low-valency ligands that interact with UBQLN2 UBA "stickers" to drive droplet disassembly (Dao et al., 2018). In contrast, we observed a substantial decrease in $T_{c p}$ at low K63-Ub4:UBQLN2 ratios, indicating a greater regime of the phase diagram over which UBQLN2 is phase separated in the presence of K63-Ub4. This effect was clearest at the lowest concentration tested $(12.5 \mu \mathrm{M})$ that approaches the physiological 
UBQLN2 concentration (1-2 $\mu \mathrm{M}$ ) (Dao et al., 2018). At $37{ }^{\circ} \mathrm{C}, 12.5 \mu \mathrm{M}$ UBQLN2 did not phase separate by itself or with Ub, K48-Ub2, K63-Ub2, and K48-Ub4, but readily phase separated with K63-Ub4 between Ub:UBQLN2 ratios of 0.3:1 ( $1 \mu \mathrm{M} \mathrm{K63-Ub4)} \mathrm{and} \mathrm{1.3:1} \mathrm{(} 4 \mu \mathrm{M} \mathrm{K63-}$ Ub4). Our data illustrate a transition from UBQLN2 LLPS driven by homotypic interactions to a system where LLPS is driven, at least partially, by heterotypic interactions between UBQLN2 and K63-Ub4 (Dao and Castañeda, 2020). Therefore, polyUb chains of different linkages can substantially alter the driving forces of phase-separating systems.

NMR titrations reveal differences in how K48- and K63-Ub4 interact with UBQLN2

To examine the molecular origins of the differential effects of K48 or K63-Ub4 on UBQLN2 LLPS, we used NMR to map the interactions between Ub4 and UBQLN2. First, we looked at FL UBQLN2 in the presence of Ub4, but the UBA resonances were severely attenuated (Fig. S3A). The large and oligomerizing FL UBQLN2 molecules can form even bigger complexes with multivalent Ub4, precluding NMR studies. To circumvent this issue, we used UBQLN2 450-624, a C-terminal construct that exhibits LLPS behavior similar to FL UBQLN2 and is amenable to NMR studies (Dao et al., 2018, 2019). To determine if UBQLN2 450-624 is suitable for studying the interactions between UBQLN2 and polyUb, we obtained temperature-composition phase diagrams using different amounts of K48 or K63-Ub4. Our phase diagrams showed that the two Ub4 similarly alter LLPS of 450-624 and FL UBQLN2 (Fig. S3B). Therefore, for subsequent NMR experiments, we collected data using UBQLN2 450-624 under non-phase separating conditions.

Upon titration of K48 or K63-Ub4 into ${ }^{15} \mathrm{~N}$-labeled UBQLN2 450-624, we observed severe broadening of UBA peaks, likely due to the large size of the Ub4:UBQLN2 450-624 complex (> 35 kDa Ub4 + 1-4X 17 kDa UBQLN2 450-624). Despite this, we could follow the resonances and observe chemical shift perturbations (CSPS) mainly for UBA residues (Fig. 2A), indicating that $\mathrm{Ub}$ units in the chains bind primarily to UBA and in a manner like monoUb. However, we also observed CSPs (Fig. 2A) and reduced peak intensity (Fig. 2B) for residues 555-570, particularly with K48-Ub4. We recapitulated the same trends with UBQLN2 487-624 (Fig. 2C). Resonances in UBQLN2 487-624 exhibit better NMR signal to noise quality since this construct does not oligomerize, undergo LLPS, or exhibit backbone dynamics on a slow millisecond timescale (Dao et al., 2018). Residues 555-570 were not perturbed in the presence of monoUb (Dao et al., 2018). Furthermore, we did not observe CSPs outside of the UBA domain in UBQLN2 450-624 construct lacking residues 551-569 (Fig 2D). Interestingly, residues 555-570, but not the surrounding regions, exhibits slight helical propensity (Dao et al., 2018). 
Together, these data indicate that the transiently helical 555-570 region either specifically interacts with K48-Ub4 or is sterically affected when K48-Ub4 binds to UBA. Since the 555-570 region is a "sticker" that mediates UBQLN2 LLPS (Dao et al., 2018), we speculated that K48Ub4 may inhibit UBQLN2 LLPS by preventing both UBA and 555-570 stickers from contributing to LLPS.
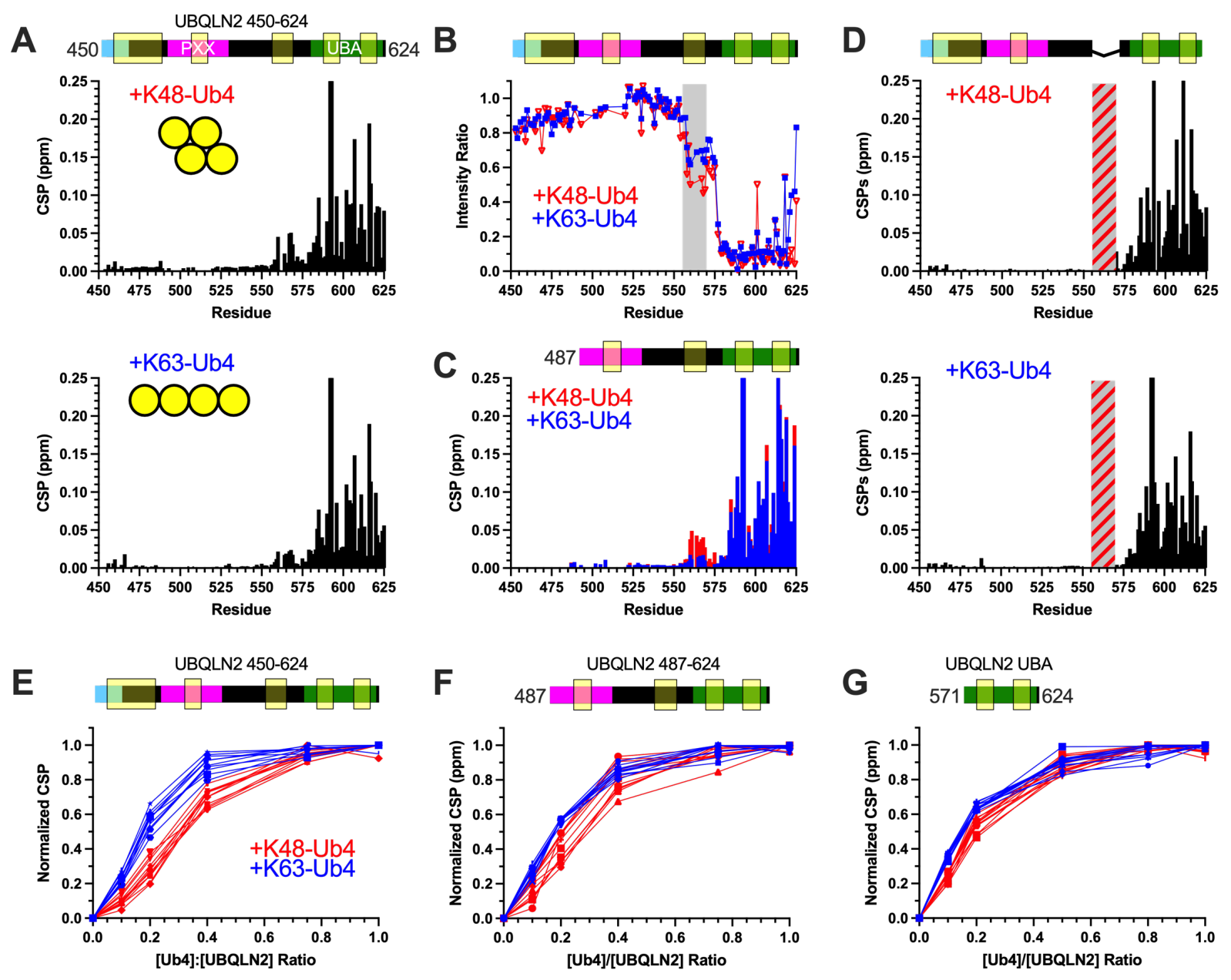

Figure 2. K48-Ub4 and K63-Ub4 bind differently to UBQLN2 450-624. (A) CSPs for residues in UBQLN2 450-624 at 1:1 ratio with Ub4, showing Ub4 interacting mainly with the UBA domain (green in the UBQLN2 domain map above) and partially with residues 555-570, especially for K48-Ub4. The highlighted regions in the domain map are important for the homotypic interactions that drive UBQLN2 LLPS (Dao et al., 2018). (B) Amide peak intensities between the unbound and Ub4-bound forms of UBQLN2 450-624 with the 555-570 region highlighted in gray. (C) CSPs for residues in UBQLN2 487-624 at 1:1 ratio with Ub4. (D) CSPs for residues in UBQLN2 450-624 lacking residues 551-569 at 1:1 ratio with Ub4. (E-G) Normalized titration curves of 11 amide resonances for UBQLN2 450-624 (E), 487-624 $(F)$ and 571-624 (G) constructs showing sigmoidal and hyperbolic behaviors in the presence of increasing K48-Ub4 and K63-Ub4, respectively. 
To determine whether the chains bind to UBQLN2 with different affinities, we collected residue-specific titration curves for UBQLN2 450-624 with K48 or K63-Ub4 (Fig. 2E). For K63Ub4, the titration curves were mostly hyperbolic, and we obtained a $K_{d}$ of $\sim 16 \mu \mathrm{M}$ using a singlesite binding model in which the ligand concentration is $4^{*} \mathrm{Ub}$ rather than Ub4 (Table S1). The $K_{d}$ is weaker than what we observed for monoUb $(\sim 3 \mu \mathrm{M})$, but CSPs suggest that each Ub in K63Ub4 interacts with UBQLN2 UBA in similar ways as monoUb (Dao et al., 2018). Unlike for K63Ub4, we observed sigmoidal curves for many UBA residues with the titration of K48-Ub4 (Fig. $2 \mathrm{E})$. We suspected this behavior is partially due to a required conformational change in K48Ub4 to accommodate binding of UBA to the Ub units (see below) and/or intermediate exchange during the NMR titration (Williamson, 2013). The sigmoidal behavior was still present but at a reduced extent when we titrated K48-Ub4 into UBQLN2 487-624 (Fig. 2F) or UBQLN2 UBA (Fig. 2G). This sigmoidal behavior precluded accurate determination of the binding affinity between UBQLN2 450-624 and K48-Ub4.

\section{The Ub-Ub interface in K48-Ub4 "opens" to accommodate UBQLN2 binding}

To further decipher the interactions between UBQLN2 450-624 and the two Ub4, we performed the reciprocal NMR titration of UBQLN2 $450-624$ into either K48 or K63-Ub4 with ${ }^{15} \mathrm{~N}$ labelled proximal Ub (Fig. 3A). First, we compared the HSQC spectrum of each proximal Ub unit to monoUb (in the absence of UBQLN2) and found significant CSPs for resonances in the proximal Ub of K48-Ub4, but not K63-Ub4, indicative of Ub-Ub interactions among K48-Ub4 subunits (Fig. 3B). The large CSPs map to the UBQLN2-binding hydrophobic patch of Ub (L8, 144, V70) and surrounding residues, consistent with known structures of K48-Ub4 (Fig. 3A, Eddins et al., 2007). Small angle x-ray scattering (SAXS) (Fig. 4B, Table S2) showed that unbound K48-Ub4 adopted a very compact conformation $\left(R_{g}=25.67 \pm 0.05 \AA\right)$, whereas K63Ub4 is extended $\left(R_{g}=32.33 \pm 0.09 \AA\right)$, consistent with prior structural studies (Tenno et al., 2004). Sedimentation velocity analytical ultracentrifugation (SV-AUC) data support these conclusions, as K48-Ub4 has a higher sedimentation coefficient and lower frictional ratio than K63-Ub4 (Fig. S4, Table S3). Together, these data suggested that the K48-Ub4 is compact in solution and that the Ub hydrophobic patches are not immediately available to bind ligands. 

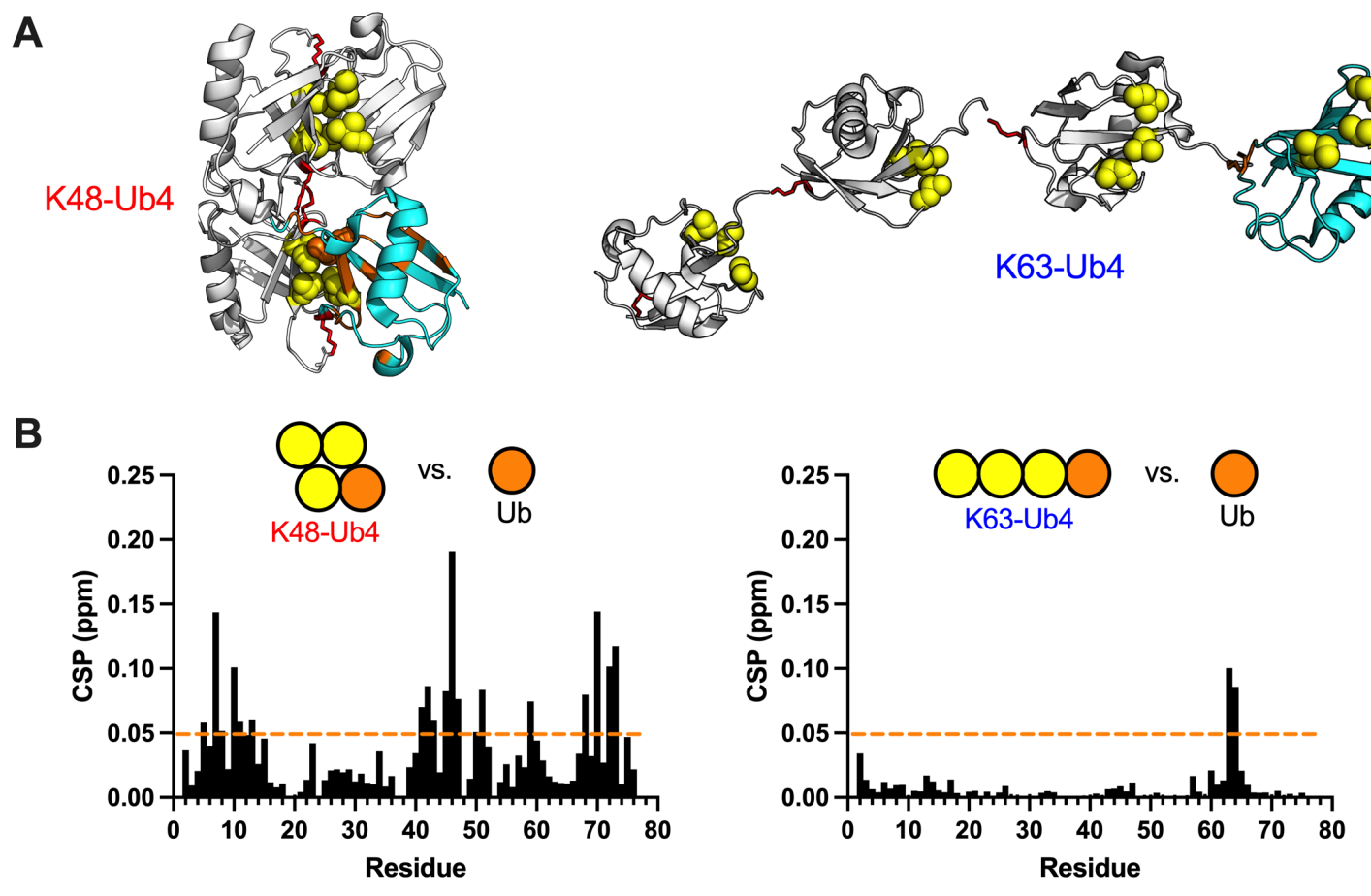

C

Prox K48-Ub4
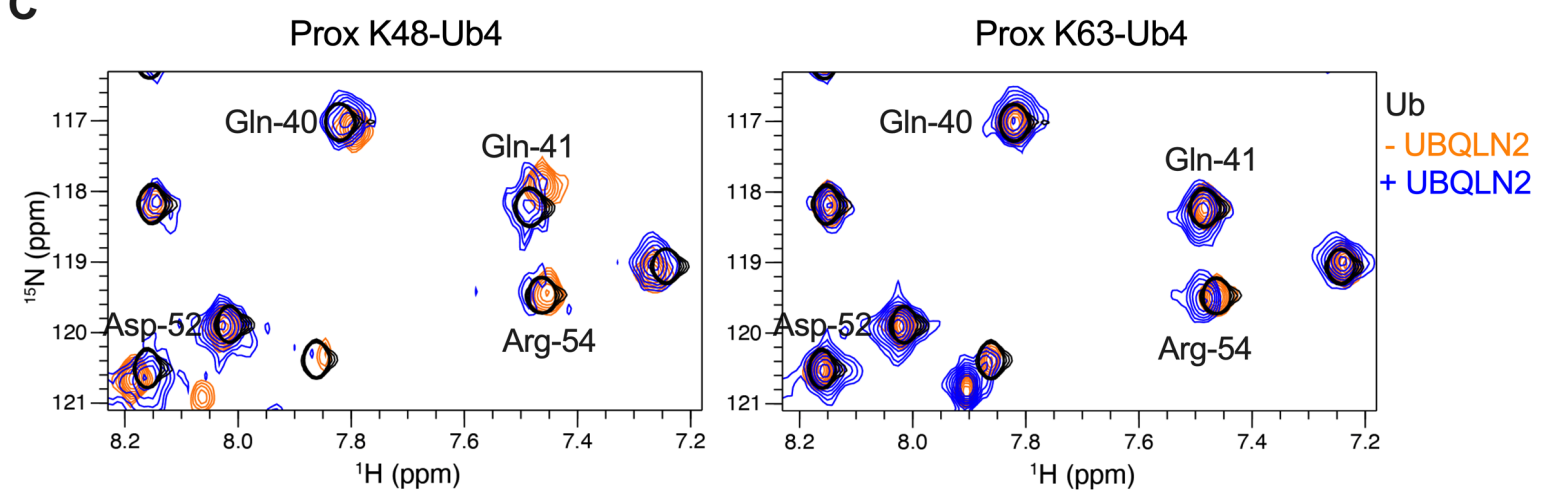

D
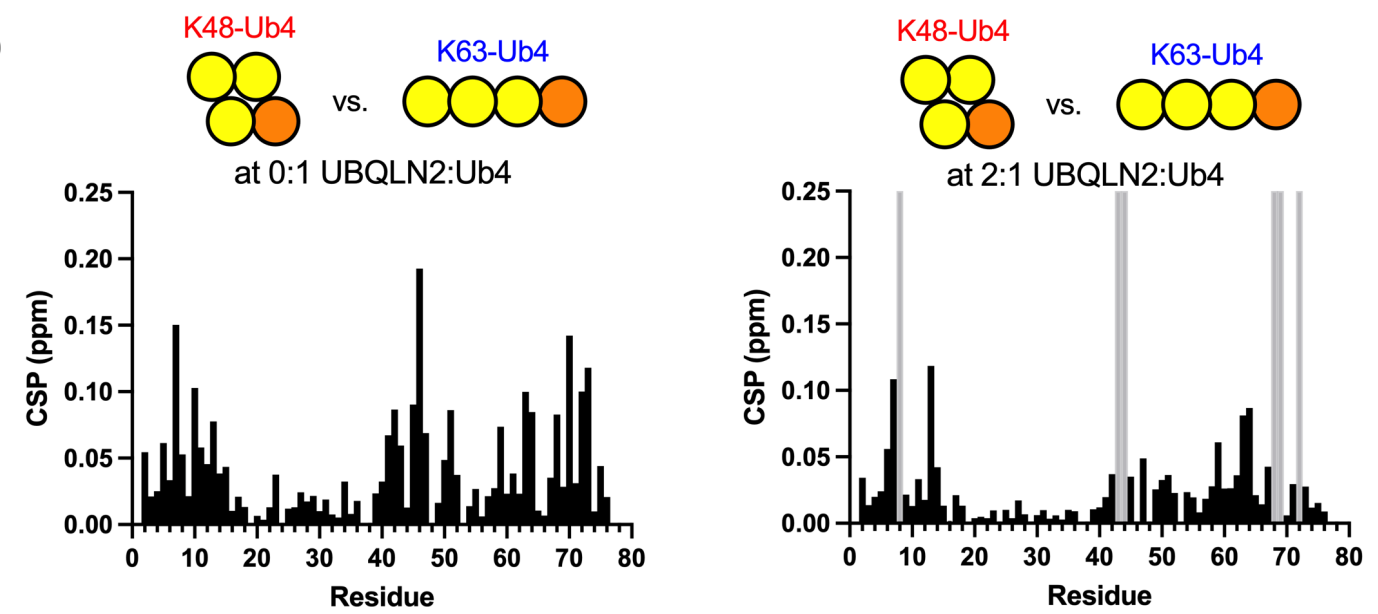

Figure 3. The Ub-Ub interface in K48-Ub4 opens to bind to UBQLN2. (A) Representative structures of K48-Ub4 (PDB ID 2O6V) and K63-Ub4 (PDB ID 3HM3). Proximal Ub unit is color-coded cyan as this unit 
was selectively ${ }^{15} \mathrm{~N}$-labeled for NMR binding experiments. Hydrophobic patch residues L8, 144, and V70 are colored yellow and shown as spheres. Red sticks indicate K48 or K63 linkage. CSPs > 0.05 ppm for proximal Ub resonances vs. monoUb positions (in the absence of UBQLN2) are color-coded orange. (B) Proximal Ub resonances in Ub4 were compared to monoUb, as noted in schematic. (C) ${ }^{15} \mathrm{~N}-{ }^{1} \mathrm{H}$ SOFASTHMQC NMR spectra of proximal Ub of K48-Ub4 or K63-Ub4 in the absence and presence of UBQLN2 450-624 at a 2:1 UBQLN2:Ub4 ratio, which corresponds to 0.5:1 UBQLN2:Ub ratio or $100 \mu \mathrm{M}: 50 \mu \mathrm{M}$ UBQLN2:Ub4. Unbound monoUb spectrum (black) is overlaid for comparison (only 5 contours plotted to denote position). NMR spectra collected and processed under identical conditions. (D) CSPs of proximal $\mathrm{Ub}$ resonances in K63-Ub4 vs. the same resonances in K48-Ub4 at 0:1 and 2:1 UBQLN2:Ub4 ratios. Gray bars indicate K48-Ub4 resonances that were too weak to be detected.

Upon titration of UBQLN2 450-624, we observed that several ${ }^{15} \mathrm{~N}$-labelled proximal Ub of K48-Ub4 resonances of residues near the Ub-Ub interface (residues 10, 39, 40, 41, 51, 52, 73, 75) converged to the locations of the same resonances in the unbound monoUb (Fig. $3 \mathrm{C}$ ), indicating that these resonances in ${ }^{15} \mathrm{~N}$-labelled proximal Ub of K48-Ub4, as UBQLN2 450-624 was titrated, were in similar chemical environments as free monoUb. In contrast, the same ${ }^{15} \mathrm{~N}$ labelled proximal Ub of K63-Ub4, in which no Ub units are in close contact with another, did not move significantly with addition of UBQLN2 450-624 (Fig. 3C). In other words, the hydrophobic patch of the proximal Ub of K48-Ub4 exhibited different chemical environments than that of Ub and the proximal Ub of K63-Ub4 in the absence of UBQLN2 450-624 but was in similar local environments in the presence of UBQLN2 450-624 (Fig. 3D). These data are consistent with our hypothesis that K48-Ub4 needs to "open" to accommodate UBA binding, based on the sigmoidal behavior of the UBQLN2 titration curves (Fig 2E). Therefore, the Ub binding patch is readily accessible in K63-Ub4 but requires an additional conformational change in K48-Ub4 to interact with UBQLN2. These observations resulted from differences in chain conformations and not binding ability as SV-AUC experiments of K48 or K63-Ub4 in the presence of UBQLN2 487624 at a 2:1 UBQLN2:Ub4 loading ratio showed complete binding between either chain with UBQLN2 (Fig. S4). The AUC data are consistent with a model where UBQLN2:K48-Ub4 complex is slightly more compact than the UBQLN2:K63-Ub4 complex (Table S3). We used monomeric UBQLN2 487-624 for this study to avoid complications from sedimentation of oligomerizing UBQLN2 450-624 in the presence of tetravalent ligands.

In summary, we suspect that K48-Ub4 does not readily promote UBQLN2 LLPS through heterotypic interactions because K48-Ub4 (1) interacts with the 555-570 "sticker" required for LLPS, (2) is compact in conformation, and (3) contains Ub hydrophobic patches that are not as readily accessible for UBQLN2 binding. In contrast, K63-Ub4 can act as a scaffold to promote 
LLPS since K63-Ub4 (1) minimally interacts with 555-570, (2) adopts an extended conformation, and (3) contains Ub hydrophobic patches that are readily accessible for UBQLN2 binding.

\section{Effects of K11, M1-Ub4 and tetrameric HOTag6-G10-Ub on UBQLN2 binding}

From the K48 and K63-Ub4 data, we hypothesized that differences in Ub4 conformation and Ub "sticker" accessibility are important determinants that drive differential effects on LLPS of UBQLN2. To test this hypothesis, we extended our study to examine how UBQLN2 interacts with other naturally occurring Ub4 chains, specifically K11 and M1 linkages, and a designed tetrameric HOTag6-G10-Ub (Fig. 4A). K11-Ub4 exhibits a compact conformation similar to K48Ub4 whereas M1-Ub4 is similar to K63-Ub4 in that it adopts an extended conformation and the Ub binding patches are readily accessible, as confirmed by SAXS scattering data (Fig. 4B, Fig. S5A). HOTag6-G10-Ub comprises HOTag6, which exists as tetramers in solution (Zhang et al., 2018), and tethered Ub, linked by 10 glycines, to form a tetrameric complex with high conformational flexibility between the Ub units (Fig. 4B, Fig. S5). Therefore, these three constructs, together with $\mathrm{K} 48$ and $\mathrm{K} 63-\mathrm{Ub} 4$, form a library of Ub tetramers with varying conformations, flexibility, and hydrophobic patch accessibility to UBQLN2 (Fig. 4A, 4B).

We used NMR spectroscopy to probe how these different polyUb chains interact with UBQLN2 450-624. We first obtained CSPs on UBQLN2 450-624 in the presence of K11, M1Ub4, or HOTag6-G10-Ub and observed the largest CSPs for UBA resonances, as expected (Fig. 4C). Strikingly, CSPs for K11-Ub4 were very similar to those for K48-Ub4, whereas CSPs for M1-Ub4 and HOTag6-G10-Ub were very similar to those for K63-Ub4. Amide resonances for residues 555-570 were more perturbed and peak intensities for residues 555-570 were more reduced in the presence of either compact K11 or K48-Ub4 chains, but not with the extended or more conformationally flexible M1-Ub4, K63-Ub4 or HOTag6-G10-Ub (Fig. 4C-4E).

Using NMR titration experiments, we measured binding affinities between UBQLN2 450624 and these chain types (Fig. 4F, Fig. S6). Unlike K48-Ub4 which is compact with occluded Ub hydrophobic patches, K11-Ub4 is compact with more exposed Ub hydrophobic patches (Fig. S7). Therefore, unlike for K48-Ub4, most of the UBQLN2 titration curves with K11-Ub4 did not exhibit sigmoidal behavior as K11-Ub4 might not need to open to accommodate UBQLN2 binding. Instead, hyperbolic titration curves in the presence of K11-Ub4, as well as M1-Ub4 and HOTag6-G10-Ub enabled accurate determination of the $K_{d}$ between these constructs and UBQLN2 450-624. Assuming single-site binding, $K_{d}$ values for UBQLN2 450-624 binding to HOTag6-G10-Ub, K11-Ub4, M1-Ub4 were 15.8 $\mu \mathrm{M}, 41.5 \mu \mathrm{M}$ and $32.5 \mu \mathrm{M}$, respectively, which are similar to those obtained for K63-Ub4 (16.2 $\mu \mathrm{M})$ (Fig. 4G, Fig. S6, Table S1). From these 
data, we predicted that K11-Ub4 may affect UBQLN2 LLPS similarly to K48-Ub4 due to CSPS for residues 555-570 and K11-Ub4's compact conformation, whereas M1-Ub4 and HOTag6G10-Ub may affect UBQLN2 LLPS similarly to K63-Ub4 due to similarities in binding affinity, extended conformation, and readily accessible Ub binding patches.

A
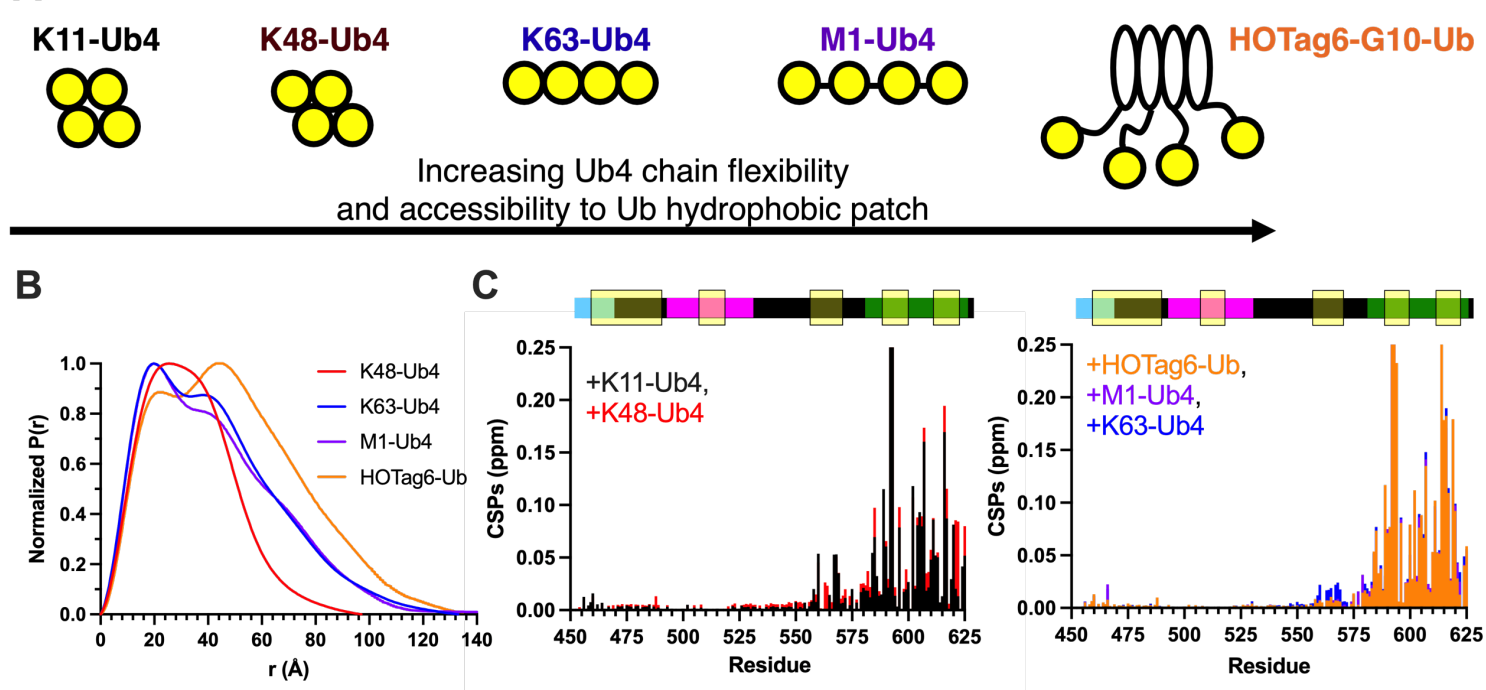

C

D
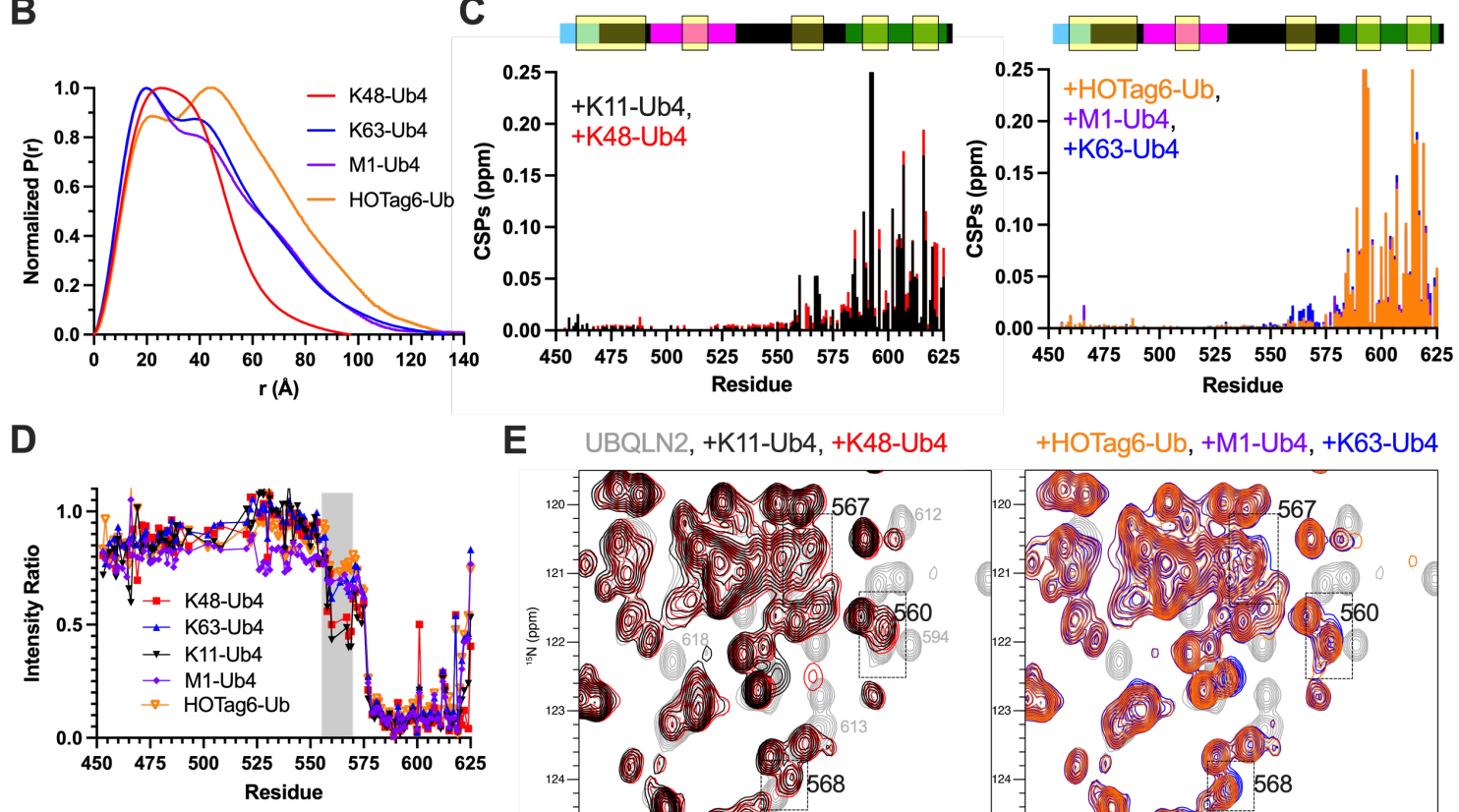

E UBQLN2, +K11-Ub4, +K48-Ub4

+HOTag6-Ub, +M1-Ub4, +K63-Ub4

F
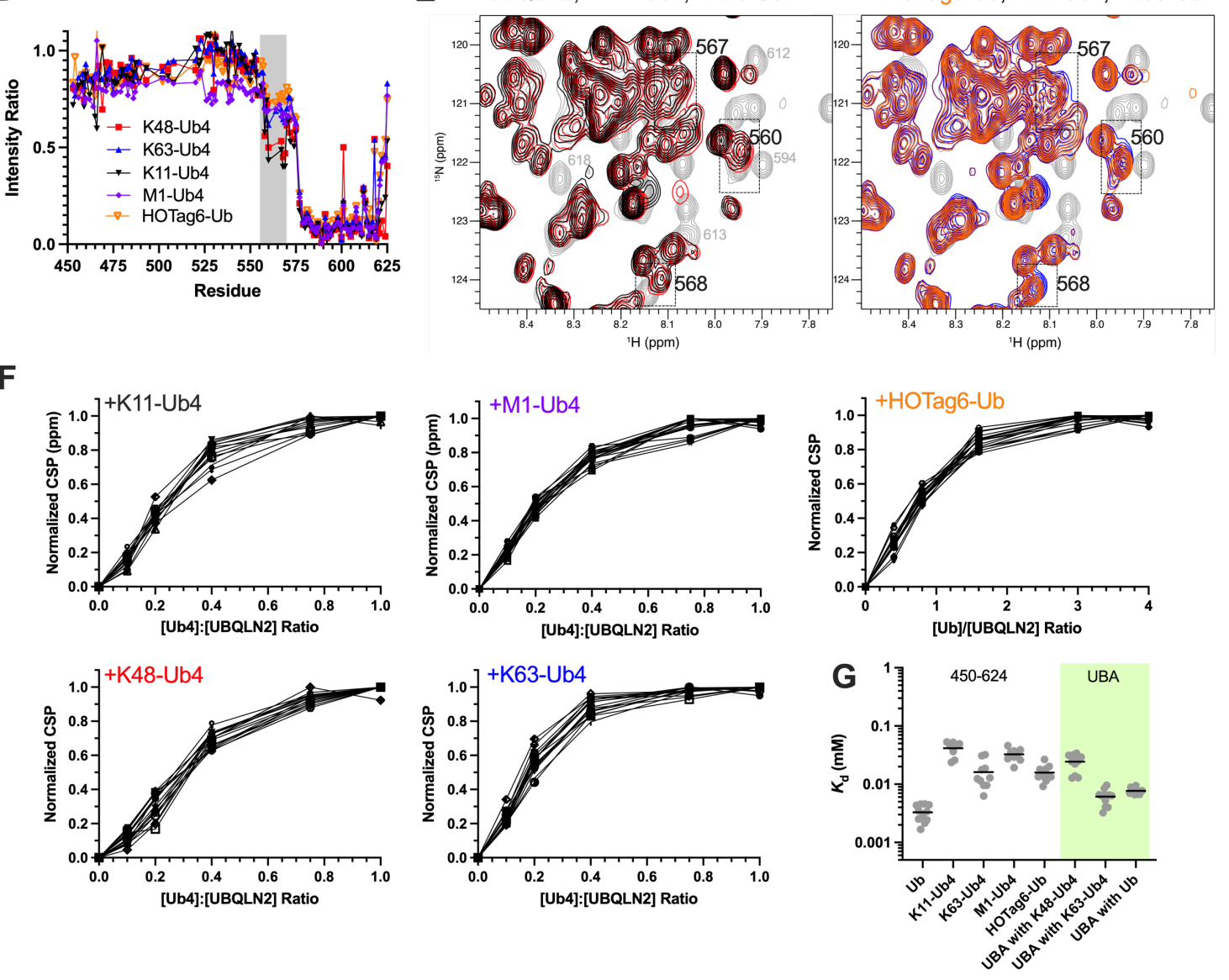
Figure 4. Compact K11 and K48-Ub4 bind UBQLN2 differently than extended K63-Ub4, M1-Ub4 and HOTag6-G10-Ub. (A) Cartoons depicting a possible solution conformation for each of the Ub4 chains studied here. (B) Normalized pair distance distribution function $P(r)$ from SAXS data for four Ub4 chain types. (C) CSPs at 1:1 Ub4:UBQLN2 for all chain types, but clustered into two categories for clarity. (D) Peak intensity comparison in the absence and presence of Ub4 at 1:1 Ub4:UBQLN2 ratio. (E) TROSYHSQC spectra of UBQLN2 450-624 in the absence and presence of different Ub4 chains. Some of the peaks in the 555-570 region are more perturbed in the presence of K11-Ub4 and K48-Ub4 but not the other chains, whereas the UBA peaks (580-624) are equally perturbed in the presence of any of the chains. (F) Normalized titration curves of UBA resonances showing sigmoidal behaviors upon titration of K48-Ub4 into UBQLN2 450-624 but hyperbolic behavior upon titration of K11-Ub4, K63-Ub4, M1-Ub4 and HOTag6-Ub. (G) Dot plot showing $K_{d}$ values for individual resonances of the UBA in 450-624 or by itself in the presence of Ub or different Ub4 chains. Black bars indicate mean.

\section{Extended and flexible, but not compact, polyUb chains promote UBQLN2 LLPS}

Next, we performed microscopy and turbidity assay experiments to determine how K11Ub4, M1-Ub4 and HOTag6-G10-Ub affect UBQLN2 LLPS. Microscopy showed that M1-Ub4 and HOTag6-G10-Ub stabilized UBQLN2 droplets over a wide range of Ub:UBQLN2 ratios (Fig. $5 \mathrm{~A}$ ), as confirmed by turbidity plots (Fig. 5B) and temperature-concentration phase diagrams (Fig. 5C). Like K48-Ub4, addition of K11-Ub4 largely disassembled UBQLN2 droplets. Strikingly, M1-Ub4 and HOTag6-G10-Ub stabilized UBQLN2 LLPS over a larger Ub:UBQLN2 range than K63-Ub4. Importantly, reentrant phase behavior still existed for all chains. As flexibility and propensity to adopt an extended conformation increased for polyUb (HOTag6-Ub > M1-Ub4 > K63-Ub4 > K48 or K11-Ub4), UBQLN2/polyUb LLPS was stabilized and promoted. These data bolster our hypothesis that more extended and flexible chains with more accessible Ub binding patch can promote UBQLN2 LLPS to a greater extent. Notably, the polyUb effect on UBQLN2 LLPS is not due to changes in binding affinities, particularly as HOTag6-G10-Ub binds UBQLN2 with a $\mathrm{K}_{d}$ similar to those for K63 and M1-Ub4 (Fig. 4G, Table S1).

To determine how polyUb affect UBQLN2 concentration in the dilute and dense phases, we labeled UBQLN2 and Ub4 with organic dyes that absorb at different wavelengths. We induced UBQLN2 LLPS with Ub4 at different Ub:UBQLN2 ratios, centrifuged to separate the two phases, and measured the absorbance of the two dyes to extract the dilute and dense phase concentrations of both UBQLN2 and Ub4 (see Methods, Fig. 5D). The UBQLN2 dilute phase concentration, or $\mathrm{C}_{\mathrm{sat}}$, was entirely consistent with our phase diagrams (Fig. 5C). For K48 Ub4, $\mathrm{C}_{\text {sat }}$ barely changed at low Ub:UBQLN2 ratios but increased to total UBQLN2 concentration (only dilute phase existed) at high ratios. Conversely, at low Ub:UBQLN2 ratios for K63, M1-Ub4 and 
HOTag6-G10-Ub, $\mathrm{C}_{\text {sat }}$ significantly decreased before increasing again at high ratios. Effectively, the change in $\mathrm{c}_{\text {sat }}$ mirrored the UBQLN2/polyUb phase diagrams, in the order of increasing chain flexibility (Fig 4A). Intriguingly, although the addition of Ub4 significantly changed the UBQLN2 dilute phase concentration, the UBQLN2 dense phase concentration remained the same in the presence of any Ub4, regardless of Ub:UBQLN2 ratios. These data suggest that the addition of polyUb chains regulates UBQLN2 LLPS by changing the volume fraction, but not concentration, of the dense phase, consistent with a previous computational study for generic systems (Ruff et al., 2021a, 2021b). Therefore, HOTag6-G10-Ub, and to lesser extents K63 and M1-Ub4, can enhance UBQLN2 LLPS without diluting the UBQLN2 dense phase concentration over a wide range of polyUb ligand concentrations.

Unlike for UBQLN2, $c_{\text {sat }}$ for Ub4 increased with increasing Ub:UBQLN2 ratios or total Ub concentrations (Fig. 5D). At low ratios, $C_{\text {sat }}$ for Ub4 were in the following order: K48-Ub4 > K63Ub4 > M1-Ub4 > HOTag6-G10-Ub, indicating that HOTag6-G10-Ub is preferentially recruited to the dense phase and K48-Ub4 is the least likely to be in the dense phase. Consistently, the Ub4 dense phase concentrations followed the opposite trend of K48-Ub4 < K63-Ub4 < M1-Ub4 < HOTag6-G10-Ub. Interestingly, like for UBQLN2, the Ub dense phase concentrations did not vary with changing Ub:UBQLN2 ratios. However, to our surprise, the Ub:UBQLN2 ratio in the dense phase is much lower than 1:1. Even for HOTag6-G10-Ub, which has the highest dense phase concentration out of the four Ub4, the Ub:UBQLN2 ratio is only about $0.4: 1$. The substoichiometric Ub:UBQLN2 ratio inside the dense phase is maintained to at least 4:1 total Ub:UBQLN2 ratio (Fig. 4D). These observations mean that, at a given point, more than half of the UBQLN2 UBA sites are still unbound, i.e. free from ligand binding, in the dense phase. We surmise that Ub-unbound UBA stickers are still needed for interactions with other UBQLN2 regions to drive LLPS. UBQLN2 is a scaffold that undergoes LLPS via homotypic interactions. Upon heterotypic interactions with highly accessible and flexible multivalent ligands via its UBA stickers, UBQLN2 LLPS can be enhanced (Fig. 7A). Hence, the interplay between homotypic and heterotypic interactions is essential for how multivalent ligands that bind to sticker sites on scaffold can modulate LLPS.

The differential partitioning of UBQLN2 and Ub4 into dilute and dense phases can also be observed by fluorescence microscopy (Fig. 5E). UBQLN2 localized mostly inside the droplets at both Ub:UBQLN2 ratios $(0.1: 1$ and $1: 1)$ for all four chains. However, at low Ub:UBQLN2 ratio (0.1:1), K63-Ub4, M1-Ub4 and HOTag6-G10-Ub were also mainly observed inside the droplets, whereas K48-Ub4 was slightly enriched in the droplets compared to outside. In contrast, at higher Ub:UBQLN2 ratio (1:1), whereas K48-Ub4 was similarly distributed in the dense and 
dilute phases and a significant fraction of K63-Ub4 was also observed in the dilute phase, M1Ub4 and HOTag6-G10-Ub were enriched inside the droplets. These qualitative observations are entirely consistent with our measured values for the dilute and dense phase concentrations, whereby HOTag6-G10-Ub is preferentially recruited to droplets.

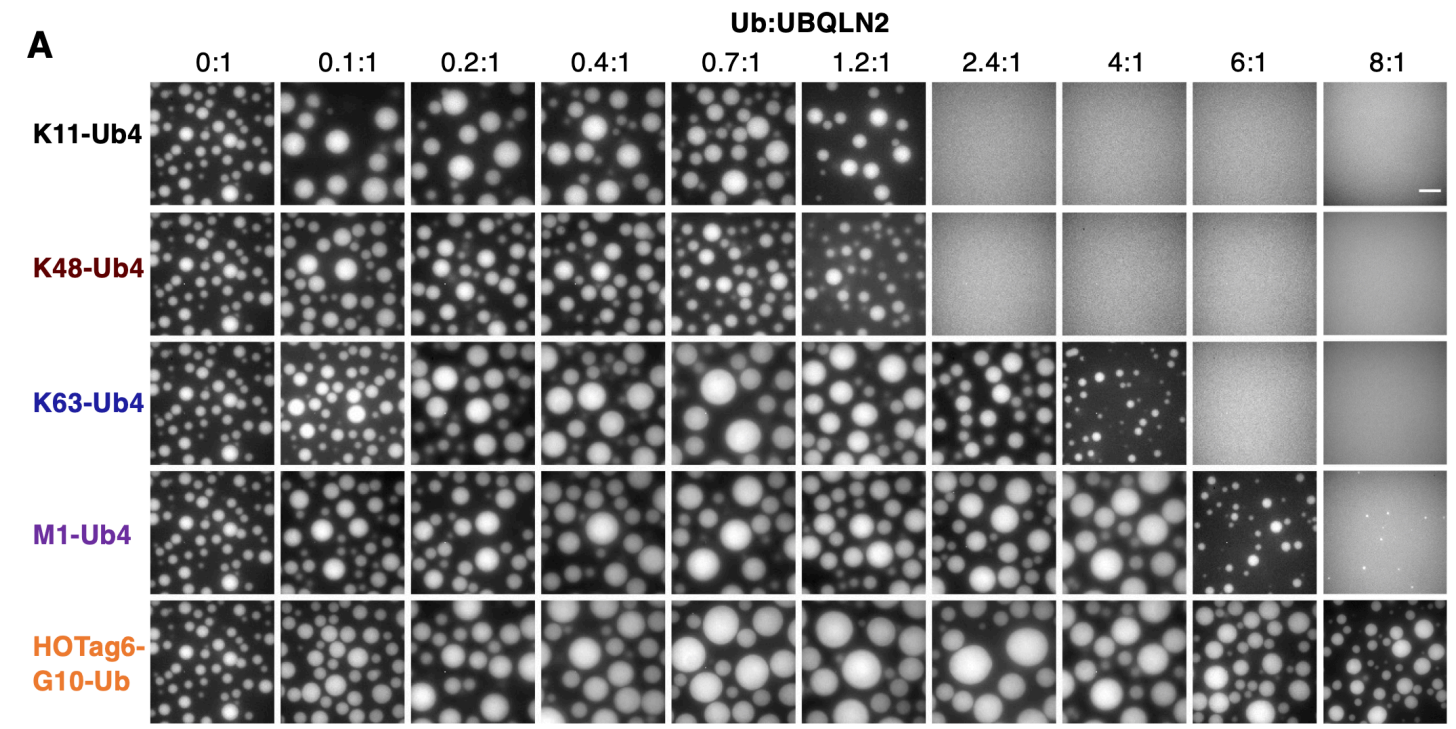

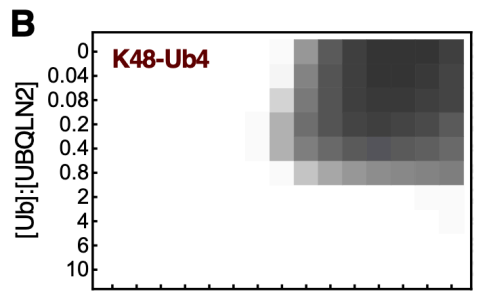
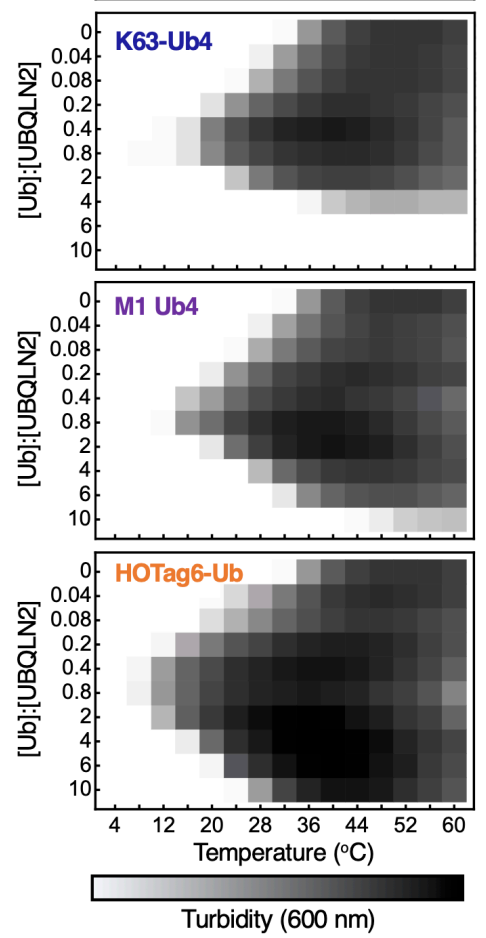

C

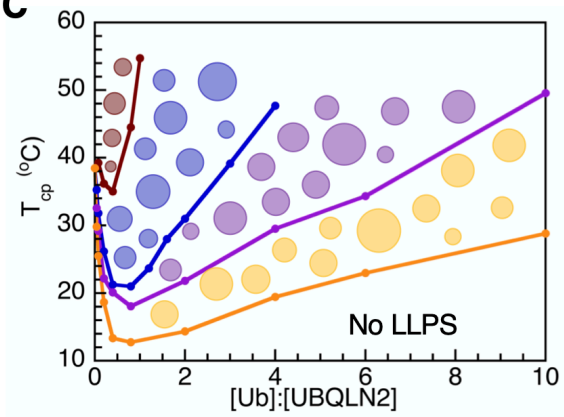

D

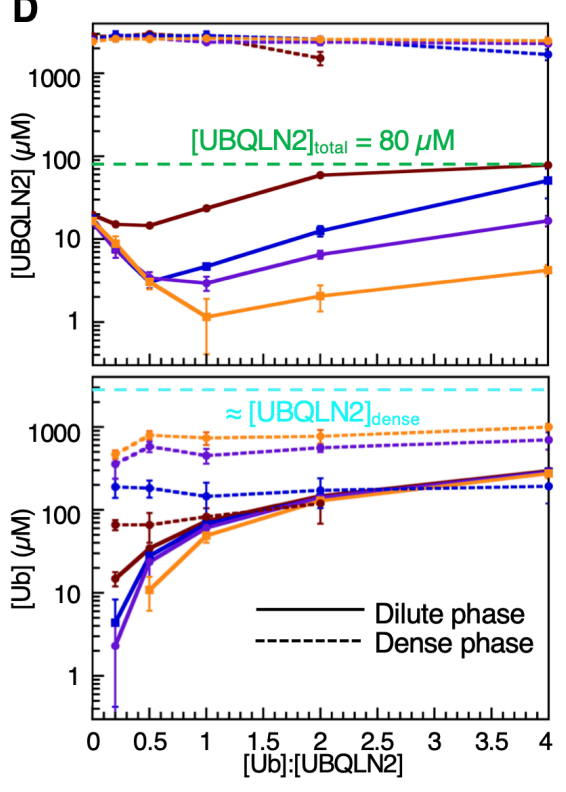

E
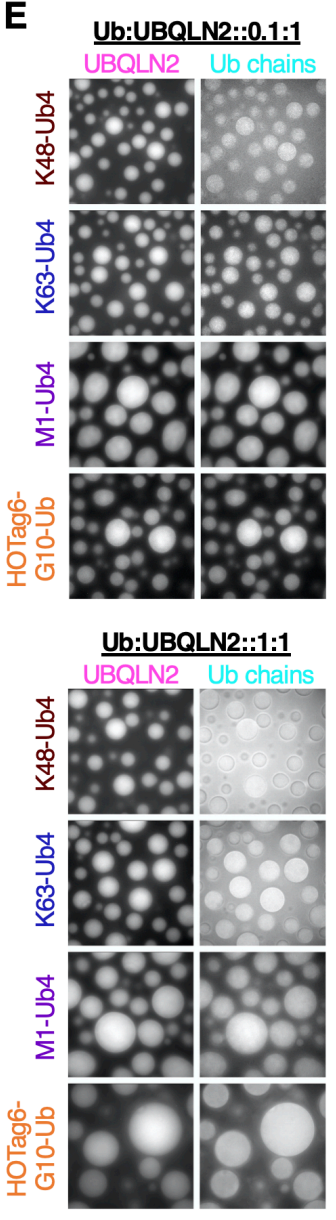
Figure 5. The effects of polyUb chains on UBQLN2 LLPS are dependent on chain flexibility I accessibility to Ub binding patch. (A) Fluorescence microscopy showing enhancement or inhibition of UBQLN2 droplet formation at increasing amounts of chains at $50 \mu \mathrm{M}$ UBQLN2 in $20 \mathrm{mM}$ NaPhosphate and $200 \mathrm{mM} \mathrm{NaCl}\left(\mathrm{pH} \mathrm{6.8)}\right.$ at $30^{\circ} \mathrm{C}$. [Ub]:[UBQLN2] reflects the ratio between $\mathrm{Ub}$ monomers and UBQLN2 molecules. Solution is spiked with Alexa Fluor 647-labeled UBQLN2. The same image is used for the $0: 1$ condition. Scale bar, $5 \mu \mathrm{m}$. (B) Representative results from spectrophotometric turbidity assay as a function of temperature comparing the effects of varying amounts K48-, K63-, and M1-Ub4 and HOTag6-G10-Ub on UBQLN2 LLPS at $30 \mu \mathrm{M}$ UBQLN2. (C) Temperature-component phase diagrams showing the changes in LCST phase transition cloud-point temperatures as a function of Ub:UBQLN2 for different Ub4 at $30 \mu \mathrm{M} U B Q L N 2$. (D) Phase diagrams showing the dense and dilute phase concentrations of UBQLN2 (top) and Ub chains (bottom) for different Ub chains. Error bars represent the standard deviation over three trials. (E) Fluorescence microscopy showing the distribution of Ub4 (spiked with Dylight 488-labeled chains) inside and outside droplets containing $50 \mu \mathrm{M}$ UBQLN2 (spiked with Alexa Fluor 647-labeled UBQLN2) at Ub4:UBQLN2 ratios of 0.1:1 and 1:1.

UBQLN2 condensates assemble during in vitro enzymatic assembly of K63-linked chains

Our data indicate that $\mathrm{Ub}$ and polyUb with different lengths and linkages can inhibit or enhance LLPS to different extents. However, in the cell, multiple Ub species, free and conjugated to substrate proteins, are in dynamic equilibrium with one another, depending on the cell cycle, cellular environment and external stimuli (Park and Ryu, 2014). To test how changes in the distribution of different Ub species affect UBQLN2 LLPS, we devised a simplified system comprising $50 \mu \mathrm{M}$ each of purified Ub and UBQLN2 (spiked with Dylight 650-labeled UBQLN2) as well as K63-specific ubiquitination machinery, including Ub-activating enzyme E1, and Ubconjugating enzymes Mms2 and GST-Ubc13 (yeast version of UBE2V2-UBE2N). In the presence of $\mathrm{MgCl}_{2}, \mathrm{ATP}, \mathrm{TCEP}$ at $37^{\circ} \mathrm{C}$, the amount of free monoUb decreased slowly over time while K63-Ub2, Ub3, Ub4, Ub5 chains were assembled as visible on the gel around 6, 9, 12 and 15 minutes, respectively (Fig. 6A, lower left). Notably, after 27 minutes, free monoUb is still the predominant $\mathrm{Ub}$ species, as could be an equilibrium state for many cell types (Kaiser et al., 2011). To complement the monitoring of Ub chain formation by gel, we also observed the mixture under a microscope. At early time points, the solution was homogeneous but after about 15 minutes, micron-sized droplets appeared and grew bigger in size and number over time (Figure 6B, Movie S1). As a negative control, no droplets were observed in that time frame without $\mathrm{Ub}$ in solution. Therefore, even in the presence of a high ratio of free monoUb to polyUb, UBQLN2 can still undergo LLPS as the ratio of different longer Ub chains increases. 
A

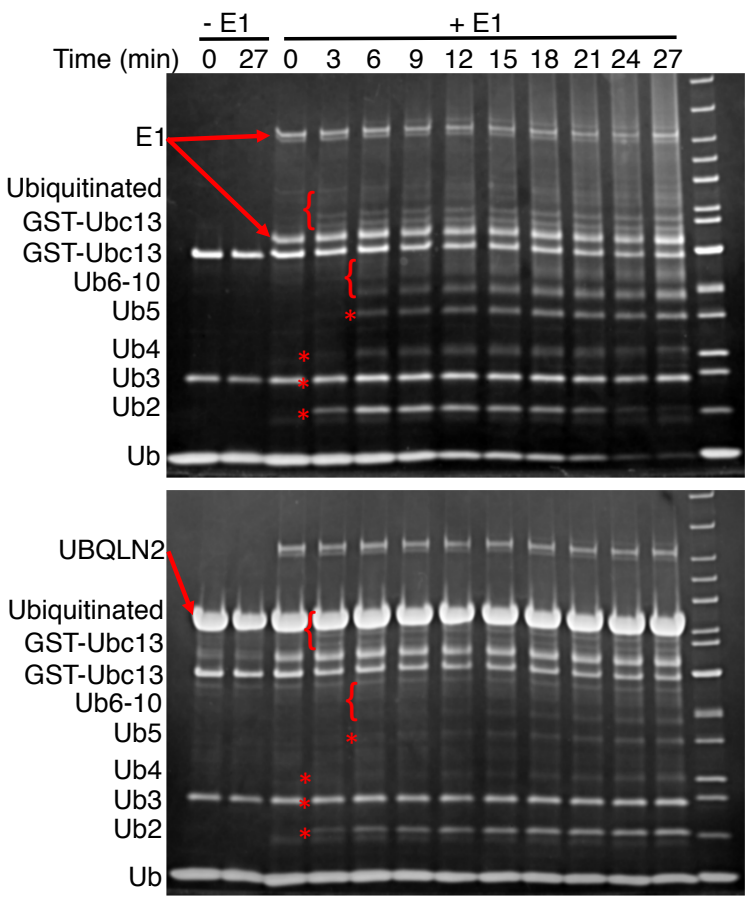

CBB R-250 (all proteins)
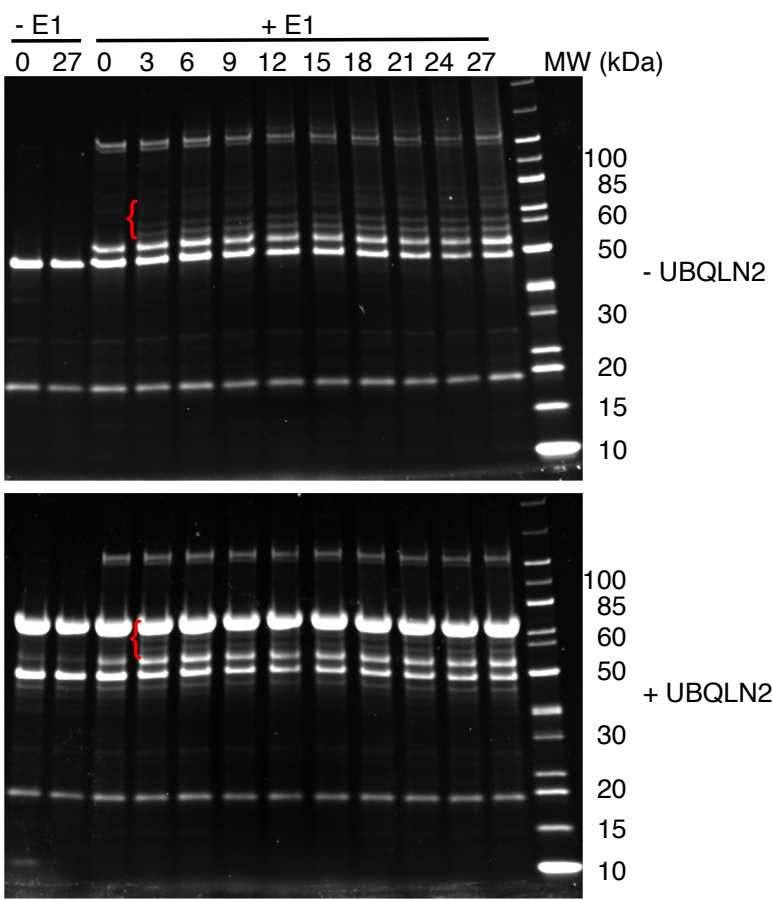

BioRad Stain-free (Proteins with W, not Ub)

B
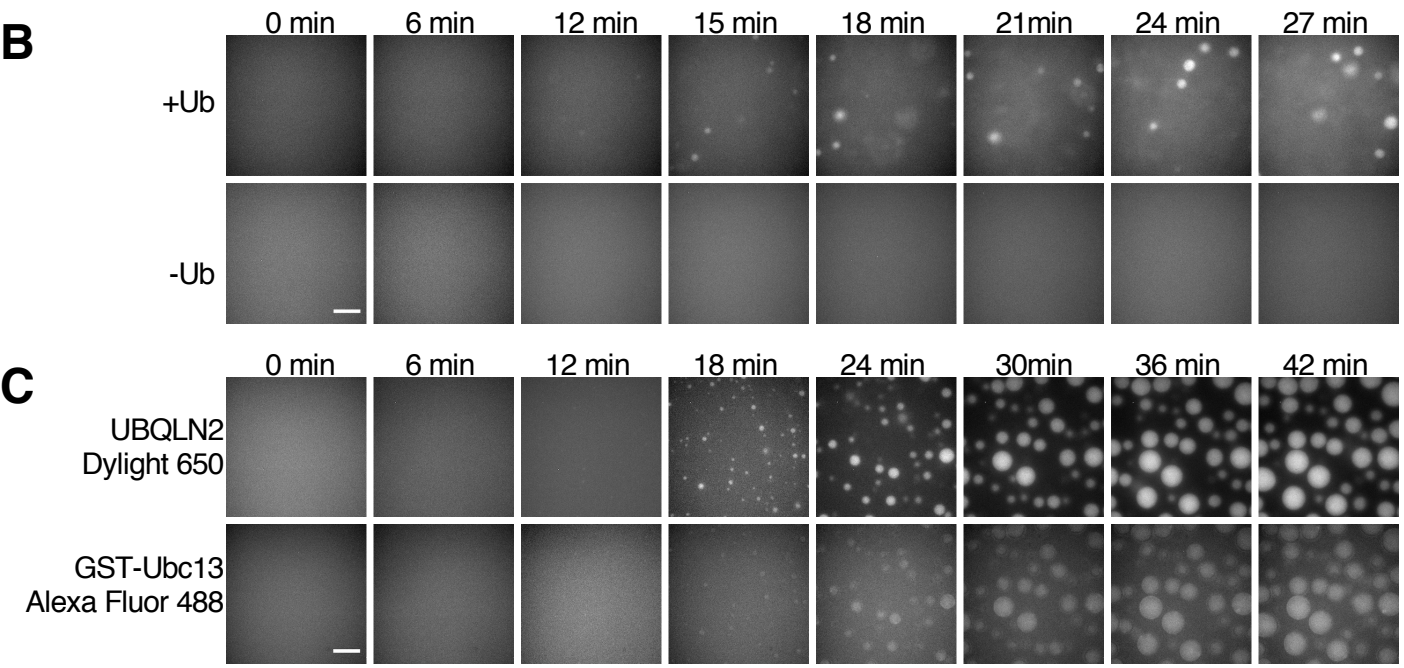

Figure 6. LLPS of UBQLN2 is induced during in vitro K63-selective ubiquitination assays. (A)

SDS-PAGE gels monitoring the formation of K63-linked polyUb chains and possibly ubiquitinated GSTUbc13 over time, as indicated by asterisks and curly brackets. K63-Ub3 migrates on the gel similarly as His-Mms2, right below $20 \mathrm{kDa}$. Experimental conditions: $\pm 50 \mu \mathrm{M}$ Ub and UBQLN2, $30 \mathrm{nM}$ Dylight 650labeled UBQLN2, $1 \mu \mathrm{M}$ mE1, $2 \mu \mathrm{M}$ His-Mms2, $4 \mu \mathrm{M}$ GST-Ubc13, 10 mM ATP and $\mathrm{MgCl}_{2}$, 3 mM TCEP, $50 \mathrm{mM}$ Tris $\mathrm{pH} 8,37^{\circ} \mathrm{C}$. The K63-linked polyUb chain reactions were done in the absence (top) and presence (bottom) of UBQLN2. Since UBQLN2 competes with E1 and E2 to bind to Ub, the presence of UBQLN2 slowed down chain formation (indicated by comparing disappearance rates of $\mathrm{Ub}$ ). The band intensity for GST-Ubc13 decreased with time. The time-dependent appearance of species between 50-85 $\mathrm{kDa}$ were obstructed in the presence of UBQLN2. These species are most likely ubiquitinated GST- 
Ubc13, and not free polyUb chains, since these bands were observed with BioRad Stain-free gels (right) that detect proteins with tryptophan residues unlike $\mathrm{Ub}$ or polyUb chains. (B) Time-lapse fluorescence microscopy monitoring UBQLN2 droplet formation in the presence of Ub and K63-selective ubiquitination machinery. Experimental conditions were the same as in (A). Imaging was done in solution above the coverslip. Scale bar, $5 \mu \mathrm{m}$. (C) Time-lapse fluorescence microscopy monitoring formation of UBQLN2and GST-Ubc13-containing droplets in the presence of $\mathrm{Ub}$ and K63-selective ubiquitination machinery. Experimental conditions were the same as in (A) with addition of $30 \mathrm{nM}$ Alexa Fluor 488-labeled GSTUbc13. Imaging was done at the coverslip surface. Scale bar, $5 \mu \mathrm{m}$.

Aside from the formation of free K63-linked polyUb chains, we also observed possible formation of ubiquitinated GST-Ubc13 as the intensity of free GST-Ubc13 reduced over time (Fig. 6A). Ub-binding proteins, like Ubc13, can be ubiquitinated in the presence of only E1 and E2 (Hoeller et al., 2007). There seemed to be several species of ubiquitinated GST-Ubc13, most likely mono-ubiquitinated at multiple lysine residues. We wondered if GST-Ubc13 could enter UBQLN2 condensates that form in the presence of K63-selective ubiquitination machinery. To test this, we set up the same experiment as above, spiked it with a small amount of Alexa Fluor 488-labelled GST-Ubc13 and observed droplet formation over time (Fig. 6C). Excitingly, we could see slight enrichment of GST-Ubc13 in UBQLN2 droplets. Most GSTUbc13 was not ubiquitinated and therefore not localized to UBQLN2 droplets. Ubiquitinated GST-Ubc13 seen here might act like a ubiquitinated substrate that can enter UBQLN2 droplets or even enhance UBQLN2 LLPS before being shuttled to the next step in a PQC pathway. Together, these data indicate that a change in the equilibrium of $\mathrm{Ub}$ species (free, conjugated monoUb and polyUb chains) in response to a certain cellular signal can affect UBQLN2 LLPS ability, and perhaps functions in PQC. 


\section{Discussion}

Recent studies have highlighted the important role of Ub and polyUb chains in modulating or driving LLPS of Ub-binding shuttle proteins hHR23B, UBQLN2 and p62 (Dao and Castañeda, 2020; Dao et al., 2018; Sun et al., 2018; Yasuda et al., 2020; Zaffagnini et al., 2018). However, these results appear to be at odds with each other. For example, K48-Ub4 disassembles UBQLN2 droplets but drives hHR23B LLPS. Long K63 chains drive p62 LLPS but are much less effective at driving hHR23B LLPS compared to K48 chains. Our mechanistic understanding of how five types of polyUb chains interact with UBQLN2 reconcile these differences and, together with other studies, offer general principles governing the driving force of polyUb chains on shuttle protein LLPS.

We showed that K48-linked chains of four or fewer Ub units generally inhibited UBQLN2 LLPS whereas K63-linked chains enhanced LLPS over a wide range of Ub:UBQLN2 ratios. We postulated that differences in either chain conformations or binding affinities among UBQLN2 and these chains lead to these different behaviors. We demonstrated, using additional K11, M1Ub4 chains and HOTag6-G10-Ub, that more extended chains with easily accessible Ub hydrophobic patches can readily promote UBQLN2 LLPS. K63- and M1-linked chains also stabilize LLPS with other proteins, such as p62 (Sun et al., 2018; Zaffagnini et al., 2018). Although we could not accurately determine the binding affinity between UBQLN2 and K48-Ub4, our NMR data indicated binding of K48-Ub4 to both FL UBQLN2 (peak attenuation upon addition of K48-Ub4) and UBQLN2 450-624. The titration curves for K48-Ub4 were saturated at about 0.8:1 K48-Ub4:UBQLN2 450-624 ratio, which is not very different from that for K63-Ub4 (Fig. 2E), indicating similar binding affinities between K48 and K63-Ub4 to UBQLN2 450-624. Moreover, although the binding affinities between UBQLN2 and HOTag6-G10-Ub, M1-Ub4, and K63-Ub4 were similar (Fig. 4G), HOTag6-G10-Ub greatly reduced csat for UBQLN2 compared to K63-Ub4. Therefore, our data suggested that binding affinities do not contribute significantly to the differences in the effects of polyUb chains on UBQLN2 LLPS.

Interestingly, pulldowns of K48- and K63-linked chains using GST-tagged UBQLN1, a close UBQLN2 homolog that also undergoes LLPS (Gerson et al., 2021), show that FL UBQLN1 only interacts with longer K63-linked chains (Harman and Monteiro, 2019). However, UBQLN1 and UBQLN2 UBAs have no significant preference for either chain (Fig. 4G) (Raasi et al., 2005; Zhang et al., 2008). We speculate that K63-, but not K48-linked, chains undergo LLPS with UBQLN1 during the pulldown process and thus appears to be a tighter binding partner. Like UBQLN2, p62 preferentially phase separates with K63- and M1-linked chains (Sun et al., 2018; Zaffagnini et al., 2018). Also like UBQLN2, FL p62 can only bind to K63-linked chains through 
pulldown assays (Cabe et al., 2018), whereas p62 UBA bind to both chains with similar affinities (Long et al., 2008; Raasi et al., 2005). Could the preferential pulldown of K63-linked chains by p62 also be an artifact of LLPS? Direct determination of binding affinities and mechanisms between p62 and these chains is needed to tease out the contributions of chain conformation and binding affinity to differences in how p62 phase separates in the presence of different chains. Unlike UBQLN2 and p62, hHR23B binds more tightly to and phase separates preferentially with K48-linked chains than K63-linked chains (Nathan et al., 2013; Raasi et al., 2005; Yasuda et al., 2020). Together, these data indicate that when binding affinities are similar, extended chains with easily accessible Ub hydrophobic patches can stabilize LLPS of Ubbinding shuttle proteins more efficiently. However, preferential binding between a shuttle protein and a compact chain can compensate for the chain's less extended structure and promote LLPS more efficiently. Moreover, our "designed Ub4", HOTag6-G ${ }_{10}-$ Ub with a long linker between HOTag6 and individual Ub units, resembles a multi-monoubiquitinated substrate. Therefore, substrates that are post-translationally modified with multi-monoubiquitination could drive phase separation of UBQLN2 and potentially other Ub-binding adaptor proteins.

Through promoting or inhibiting LLPS, polyUb chains could be potent modulators of signaling outcomes in the cell, akin to PARylation, another PTM that regulates LLPS via length and branching (Reber and Mangerich, 2021). For example, both K11- and K48-linked chains are linked to proteasomal degradation pathways, with a branched K11/K48 chain binding more tightly to the proteasome (Boughton et al., 2019). Intriguingly, only these chain types destabilize UBQLN2 LLPS. In contrast, K63- and M1-linked polyUb are typically involved in non-proteolytic signaling pathways such as cargo sorting, DNA repair, NF-K $\beta$ signaling, and autophagy (Haglund and Dikic, 2012; Linares et al., 2013; Olzmann et al., 2007; Piper et al., 2014; Sun et al., 2018). UBQLN2 has known affiliations with the proteasome, autophagy receptors (LC3) and cargo sorting adaptor proteins (epsin 1 and 2) (Lin et al.; Zheng et al., 2020). We showed that UBQLN2 LLPS can be rapidly modulated by changes in cellular signaling (e.g. formation of K63linked chains or ubiquitinated substrates, Fig. 6). The UBQLN2 concentration in cells could be below the threshold needed for LLPS such that UBQLN2 is diffuse under normal conditions. In response a cellular event, such as stress, substrates can be ubiquitinated and elicit different responses from UBQLN2, depending on the types of ubiquitination (Fig. 7). UBQLN2 can bind to K11- or K48-linked ubiquitinated substrates, stay diffuse, and shuttle substrates to the proteasome for degradation. UBQLN2 can bind to K63- or M1-linked substrates, colocalize to or form substrate-containing condensates. These condensates can be similar to p62- or hHR23Bcontaining nuclear degradation foci to which proteasomes are recruited (Fu et al., 2021; Yasuda 
et al., 2020) or initiation centers for the formation of autophagosomes (Sun et al., 2018; Turco et al., 2019; Zaffagnini et al., 2018). The polyUb effects on UBQLN2 LLPS may be the general mechanism regulating the roles of UBQLN2 and other Ub-binding shuttle proteins in the cell.

UBQLN2 is also recruited to stress granules, most likely due to its LLPS properties (Alexander et al., 2018; Dao et al., 2018). UBQLN2 expression levels are negatively correlated to the number and sizes of stress granules (Alexander et al., 2018), indicating a role for UBQLN2 in regulating the disassembly of stress granules. Stress granules are cleared either following the removal of K63-linked polyubiquitinated G3BP1, a stress granule core protein, or by autophagy, depending on the stressor types and length of exposure (Buchan et al., 2013; Gwon et al., 2021). Through differential modulation of UBQLN2 LLPS by different polyUb chains, UBQLN2 may facilitate these different stress granule clearance processes (Fig. 7). Persistent stress granules can lead to the formation of disease-linked protein-containing inclusions (Nedelsky and Taylor, 2019), that comprise, among others, PQC components such as UBQLN2 and polyUb chains (Ceballos-Diaz et al., 2015; Deng et al., 2011; Sharkey et al., 2020). Therefore, knowledge of how polyUb chains affect UBQLN2 in stress granules is essential to understanding the cellular functions of UBQLN2.

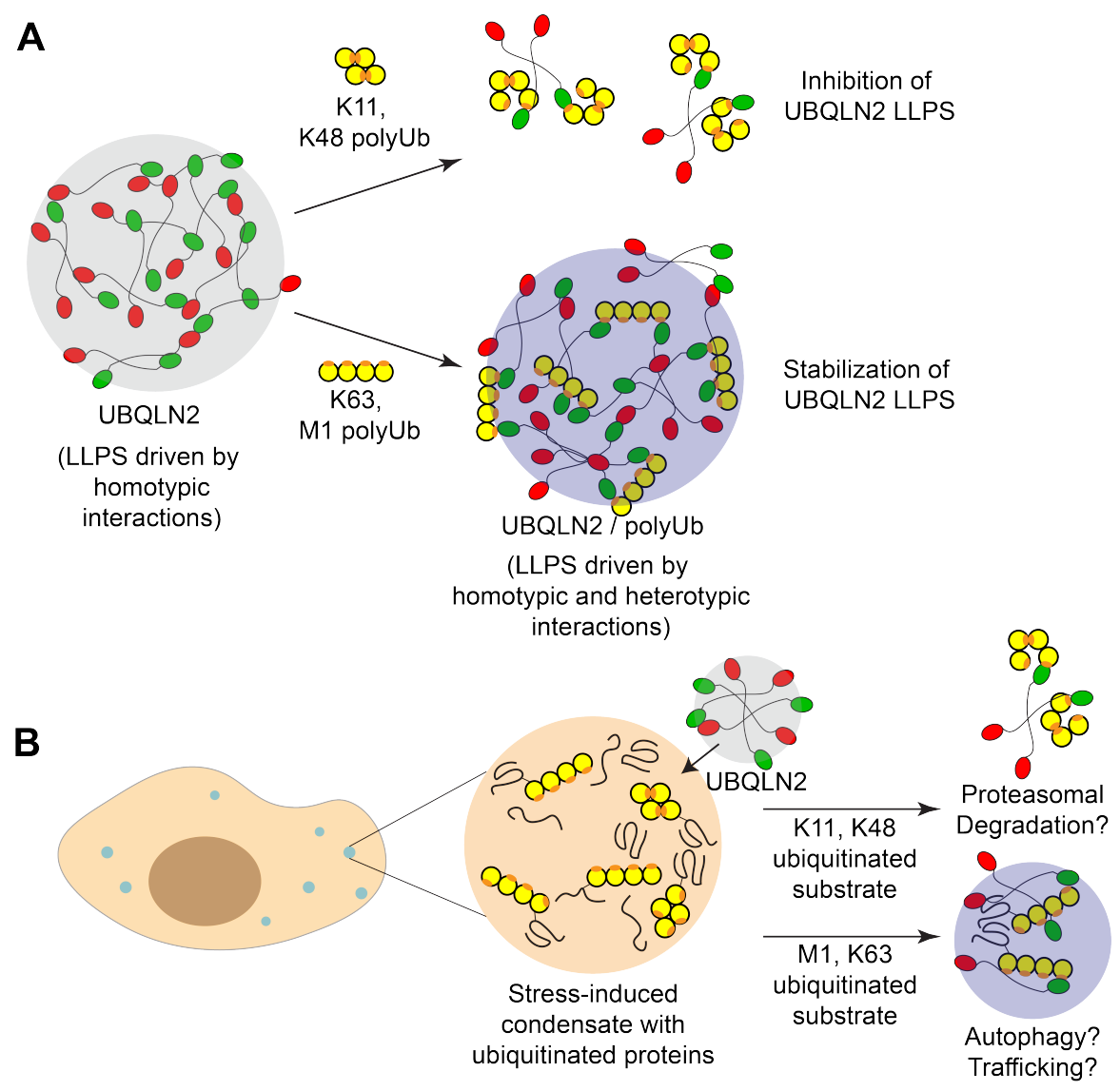


Figure 7. PolyUb chains either inhibit or stabilize LLPS via heterotypic interactions. (A) Compact polyUb chains (e.g. K11, K48) generally inhibit UBQLN2 LLPS, whereas extended chains with accessible hydrophobic patches promote heterotypic LLPS. Orange hotspots on Ub units indicate hydrophobic patch. (B) Model for how polyUb-modulated effects on LLPS may drive different cellular outcomes and signal different PQC pathways for ubiquitinated proteins in stress-induced condensates. K11 and K48 chains typically signal for proteasomal degradation, whereas extended K63 and M1 chains are implicated in autophagy and other non-proteolytic pathways.

Aside from cellular implications, our work presented here also contributes to the important question of how binding to multivalent ligands modulates the phase boundaries of phase-separating systems. Using monovalent and divalent ligands, Ruff and colleagues demonstrated through simulations that high-valency ligands that bind more tightly to spacer sites (residues or protein regions not involved in driving LLPS) are more effective at lowering the $\mathrm{C}_{\text {sat }}$ necessary for LLPS of multivalent scaffolds (Ruff et al., 2021a). Conversely, ligands that bind to sticker sites generally increase $\mathrm{c}_{\text {sat }}$, but the effect is lessened with increasing ligand valency. Our experimental work recapitulates many of these observations and offers additional insights. We systematically and quantitatively demonstrated that longer polyUb could either less effectively inhibit (K48 chains) or more effectively promote (K63 chains) UBQLN2 LLPS (Fig. 1). In fact, K48-Ub4 slightly promoted UBQLN2 LLPS at low Ub:UBQLN2 ratios. Following this trend, K48 chains with five or more Ub units would further enhance UBQLN2 LLPS, in similar manner as K63 chains with 3 or 4 Ub units. K48 and K63 polyUb ligands interact mainly with UBQLN2 UBA stickers, but K48-linked chains also interact with an additional sticker site. Therefore, K48 chains need to have increased valency (by increasing chain length) to compensate for the loss of a sticker site important for UBQLN2 self-interactions and LLPS. We expect multivalent ligands that bind to sticker sites of other systems to enhance LLPS when a certain ligand valency is reached.

Despite having the same valency and binding to the same UBQLN2 stickers (Fig. 4C) with similar affinities (Fig. 4G), the ligands K63-Ub4, M1-Ub4 and HOTag6-G10-Ub lower $\mathrm{C}_{\text {sat }}$ for UBQLN2 LLPS to highly different extents (Fig. 5D). The major difference among these chains is how the UBQLN2 binding surface is presented (i.e. the spacer length and flexibility between the stickers). Therefore, how a multivalent ligand modulates LLPS of a scaffold (e.g. UBQLN2) depends not only on binding affinities, ligand valencies, and binding sites on scaffold, but also on the properties of the spacers between the stickers on the ligand. In our case, it appears that longer, more flexible spacers are better at promoting LLPS, likely due to the ligand generating a broader interacting network. However, there must be a limit after which the multivalent ligand 
begins to behave like individual units of monovalent ligands. It would be interesting to determine what that limit is in terms of spacer length / flexibility and if this principle can be generalized to other systems.

Overall, we uncovered the molecular mechanisms enabling distinct polyUb chains to be potent regulators of phase separation of UBQLN2 and possibly other Ub-binding shuttle proteins. With this knowledge, we can design specific experiments in cells, such as inhibition of certain polyUb chains or PQC pathways, to elicit how polyUb chains may tune UBQLN2's ability to form or be recruited into condensates and then carry out PQC functions. Moreover, disease mutations in UBQLN2 have been shown to disrupt UBQLN2's roles in PQC (Chen et al., 2018; Fecto et al., 2013; Halloran et al., 2020; Hjerpe et al., 2016). Therefore, determining the effects of polyUb chains on the phase separation of UBQLN2 variants is an essential next step to elucidating disease-related mechanisms involving UBQLN2.

\section{Acknowledgements}

This work was supported by ALS Association grant 18-IIP-400, NIH R01 GM136946 (turbidity assays, microscopy, and NMR experiments) and NSF CAREER MCB 1750462 (SEC-MALSSAXS experiments) to C.A.C. NMR data were acquired on an $800 \mathrm{MHz}$ NMR spectrometer funded by NIH shared instrumentation grant 1S10OD012254. This research used resources of the Advanced Photon Source, a U.S. Department of Energy (DOE) Office of Science User Facility operated for the DOE Office of Science by Argonne National Laboratory under Contract No. DE-AC02-06CH11357. This project was supported by grant P30 GM138395 from the National Institute of General Medical Sciences of the National Institutes of Health. Use of the Pilatus 3 1M detector was provided by grant 1S100D018090-01 from NIGMS. We thank Rohit Pappu, Kiersten Ruff, Tanja Mittag, Daniel Kraut, Elliot Dine, and Susan Krueger for stimulating discussions over the years leading to this project. We also thank Ashley Canning with assistance on AUC experiments. The content is solely the responsibility of the authors and does not necessarily reflect the official views of the National Institutes of Health.

\section{Author Contributions}

Conceptualization, T.P.D. and C.A.C.; Methodology, T.P.D., Y.Y., J.H., C.A.C.; Investigation, T.P.D., Y.Y., M.S.C., W.M.; Writing - Original Draft, T.P.D., C.A.C.; Writing - Review \& Editing, T.P.D., Y.Y., M.S.C., C.A.C; Funding Acquisition, C.A.C.; Resources, C.A.C., M.S.C., J.H., W.M.; Supervision, C.A.C. 


\section{Methods}

Gene synthesis and site-directed mutagenesis

The HOTag6-G10-Ub polypeptide has the following sequence: MTLREIEELLRKIIEDSVRSVA ELEDIEKWLKKIGGGGGGGGGGMQIFVKTLTGKTITLEVEPSDTIENVKAKIQDKEGIPPDQQRL IFAGKQLEDGRTLSDYNIQKESTLHLVLRLRGG. The genes encoding HOTag6-G10-Ub and M1-Ub4-His6 were codon-optimized, synthesized and cloned into pET24b (Novagen) by GenScript (NJ, USA). K48R, K63R, K11/63R Ub and Ub-V-His6 were made using Phusion SiteDirected Mutagenesis Kit (Thermo Scientific).

\section{Protein Expression, and Purification}

Wildtype, K48R, K63R, Ub and Ub-V-His6 were expressed and purified as detailed elsewhere (Beal et al., 1996; Castañeda et al., 2016b). The gene encoding mouse E1 was a kind gift from Jorge Eduardo Azevedo (Addgene plasmid 32534, (Carvalho et al., 2011)). E1, Mms2, Yuh1 and GST-Ubc13 were expressed in Escherichia coli NiCo21 (DE3) cells in Luria-Bertani (LB) broth at $16{ }^{\circ} \mathrm{C}$ overnight. GST-E2-25K in pGEX-4T2 and GST-Ube2s-UBD in pGEX6P1 were expressed in Escherichia coli Rosetta 2 (DE3) pLysS cells in Luria-Bertani (LB) broth at $16{ }^{\circ} \mathrm{C}$ overnight. Bacteria were pelleted, frozen, then lysed via freeze/thaw method in $50 \mathrm{mM}$ Tris, 1 mM EDTA (pH 8), $1 \mathrm{mM}$ PMSF, $1 \mathrm{mM} \mathrm{MgCl}_{2}$, and $25 \mathrm{U}$ of Pierce universal nuclease. Yuh1, E1 and Mms2 were purified via $\mathrm{Ni}^{2+}$ affinity chromatography. GST-E2-25K, GST-Ubc13, and GSTUbe2s-UBD were purified via GST chromatography. All proteins were concentrated, buffer exchanged into $50 \mathrm{mM}$ Tris and $1 \mathrm{mM}$ EDTA $(\mathrm{pH} 8)$, and stored at $80^{\circ} \mathrm{C}$ for subsequent use in the production of K48- and K63-linked polyUb chains, and K11-linked Ub4.

K48-linked and K63-linked Ub2, Ub3 and Ub4 were synthesized sequentially. Briefly, equal amounts of K48R (K63R) Ub and Ub-V-His6 incubated with $1000 \mathrm{nM} \mathrm{E1}$ and $10 \mu \mathrm{M}$ GSTE2-25K (2 $\mu \mathrm{M}$ His-Mms2 and $4 \mu \mathrm{M}$ GST-Ubc13) in the presence of $10 \mathrm{mM}$ ATP, $0.3 \mathrm{mM}$ TCEP in Tris buffer at $\mathrm{pH} 8$ for 3 hours at $37^{\circ} \mathrm{C}$. This procedure generates $\mathrm{K} 48 \mathrm{R}$ (K63R) Ub2 with the C-terminal end of the proximal Ub blocked by V-His6. Yuh1 was added to remove the V-His6 from the end of Ub2, which was then purified via cation exchange column using $50 \mathrm{mM}$ ammonium acetate $(\mathrm{pH} 4.5)$ as the buffer. Protein was eluted via a linear gradient from 0 to $100 \%$ of $50 \mathrm{mM}$ ammonium acetate, $1 \mathrm{M} \mathrm{NaCl}(\mathrm{pH} 4.5)$. Purified Ub2 was then buffer exchanged into $50 \mathrm{mM}$ Tris buffer at $\mathrm{pH}$ 8. K48 and K63-Ub3 were made the same way Ub2 was made. K48 and K63-Ub4 required an additional purification step via size exclusion chromatography using a Superdex 75 HiLoad 16/600 column (GE Healthcare). K11-Ub2 was 
made with K63R as described by (Bremm et al., 2010) using mE1 and GST-Ube2s-UBD in similar conditions as for K48 and K63 chains. K11-Ub4 was made from K11-Ub2 and purified by size exclusion chromatography using a Superdex 75 HiLoad 16/600 column (GE Healthcare). The yield for K11-Ub4 was about 10\%, much lower than for K48 and K63-Ub4, which was about $50 \%$.

HOTag6-G10-Ub and M1-Ub4-His6 were expressed in Escherichia coli NiCo21 (DE3) cells in Luria-Bertani (LB) broth at $37^{\circ} \mathrm{C}$ overnight. Bacteria were pelleted, frozen, then lysed via freeze/thaw method in $50 \mathrm{mM}$ Tris, $1 \mathrm{mM}$ EDTA ( $\mathrm{pH}$ 8), $1 \mathrm{mM} \mathrm{PMSF,} 1 \mathrm{mM} \mathrm{MgCl}_{2}$, and $25 \mathrm{U}$ of Pierce universal nuclease. The cleared lysate for HOTag6-G10-Ub was then loaded onto anion exchange column and eluted with a gradient between $20 \mathrm{mM}$ HEPES, $0.02 \% \mathrm{NaN}_{3}(\mathrm{pH} 7)$ and 20 mM HEPES ( $\mathrm{pH} 7$ ), $1 \mathrm{M} \mathrm{NaCl}, 0.02 \% \mathrm{NaN}_{3}$. Fractions containing HOTag6-G10-Ub were dialyzed, loaded onto a cation exchange column, and eluted with a gradient between $50 \mathrm{mM}$ ammonium acetate ( $\mathrm{pH}$ 4.5) and $50 \mathrm{mM}$ ammonium acetate, $1 \mathrm{M} \mathrm{NaCl}(\mathrm{pH} 4.5)$. Purified HOTag6-G10-Ub was then concentrated, and buffer exchanged into $20 \mathrm{mM}$ NaPhosphate, 0.5 mM EDTA, $0.1 \mathrm{mM}$ TCEP, $0.02 \% \mathrm{NaN}_{3}$ (pH 6.8). M1-Ub4-His6 was purified via $\mathrm{Ni}^{2+}$ chromatography, dialyzed into $50 \mathrm{mM}$ Tris, $1 \mathrm{mM}$ DTT before the addition of Yuh1 to cleave the His-tag after the last Ub unit. The cleaved M1-Ub4 was then dialyzed into $50 \mathrm{mM}$ ammonium acetate $\mathrm{pH} 4.5$, loaded onto cation exchange column, and eluted with a gradient between 50 $\mathrm{mM}$ ammonium acetate $(\mathrm{pH} 4.5)$ and $50 \mathrm{mM}$ ammonium acetate, $1 \mathrm{M} \mathrm{NaCl}(\mathrm{pH}$ 4.5). The fractions containing M1-Ub4 were concentrated and exchanged into $20 \mathrm{mM}$ NaPhosphate, 0.5 $\mathrm{mM}$ EDTA, $0.1 \mathrm{mM}$ TCEP, $0.02 \% \mathrm{NaN}_{3}\left(\mathrm{pH}\right.$ 6.8). Purified proteins were frozen at $-80^{\circ} \mathrm{C}$.

Full-length UBQLN2 and UBQLN2 450-624 were expressed and purified as described by previously (Dao et al., 2018). Briefly, the constructs were expressed in E. coli Rosetta 2 (DE3) pLysS cells in Luria-Bertani (LB) broth at $37^{\circ} \mathrm{C}$ overnight. Bacteria were pelleted, frozen, lysed, then purified via a "salting out" process. $\mathrm{NaCl}$ was added to the cleared lysate to the final concentration of $0.5 \mathrm{M}-1 \mathrm{M}$. UBQLN2 droplets were pelleted and then resuspended in $6 \mathrm{M}$ urea, $20 \mathrm{mM}$ NaPhosphate, $0.5 \mathrm{mM}$ EDTA, $0.1 \mathrm{mM}$ TCEP, $0.02 \% \mathrm{NaN}_{3}(\mathrm{pH}$ 6.8). Leftover $\mathrm{NaCl}$ and urea were removed through HiTrap desalting column (GE Healthcare). Purified proteins were frozen at $-80^{\circ} \mathrm{C}$.

\section{Fluorescent Labeling}

UBQLN2, GST-Ubc13, K48-Ub4, K63-Ub4, M1-Ub4, HOTag6-G10-Ub constructs were fluorescently labelled with Dylight-488, DyLight-650 or Alexa Fluor-488 NHS Ester (Thermo Scientific), respectively, according to the manufacturer's instructions. Excess dye was removed 
via size exclusion chromatography using an ENrich ${ }^{\text {TM }}$ SEC 65010 x 300 Column (BioRad).

\section{Spectrophotometric Absorbance/Turbidity Measurements}

Protein samples were prepared by mixing protein solution containing UBQLN2 and polyUb (the concentrations of the protein stock solutions were doubled compared with the sample concentrations) and cold sodium phosphate buffer (pH 6.8, $20 \mathrm{mM}$ NaPhosphate, $0.5 \mathrm{mM}$ EDTA, $0.1 \mathrm{mM}$ TCEP, $0.02 \% \mathrm{NaN}_{3}$ ) containing $400 \mathrm{mM} \mathrm{NaCl}$ at $1: 1$ stoichiometry. The protein concentrations were chosen to cover as wide a range as possible to allow observation of phase separation during the temperature ramps. All the solutions were kept on ice for at least 5 minutes before mixing. Absorbance at $600 \mathrm{~nm}$ was recorded as a function of temperature by a Cary 3500 UV-Vis spectrophotometer using a temperature ramp rate of $1^{\circ} \mathrm{C} / \mathrm{min}$ increasing from $16{ }^{\circ} \mathrm{C}$ to $60{ }^{\circ} \mathrm{C}\left(4^{\circ} \mathrm{C}\right.$ to $60^{\circ} \mathrm{C}$ range was screened in some cases). Net absorbance values were recorded after subtracting the absorbance value of a buffer control. At least two trials $(n=$ 2) were conducted to ensure reproducibility. Data were plotted using Mathematica (Wolfram Research).

\section{Phase Diagram Measurements}

For the LCST (lower critical solution temperature) phase transition, i.e. mapping the phase boundary as temperature is increased, protein samples were prepared as described for the turbidity measurements. Cloud point temperatures were determined by fitting a Four Parameter Logistic Regression model to the data:

$$
y=d+\frac{a-d}{1+\left(\frac{x}{c}\right)^{b}} \text { (Equation 1) }
$$

Cloud point temperatures ( $\left.T_{\mathrm{cp}}\right)$ used were the points of inflection (c). Cloud point temperatures were used to define the coexistence curve as a function of ligand:protein ratio (ligand refers to polyUb chains and protein refers to UBQLN2). Fitting and plotting of data were done with Mathematica (Wolfram Research) and Kaleidagraph (Synergy Software).

\section{Bright-field Imaging of Phase Separation}

Samples were prepared to contain $50 \mu \mathrm{M}$ UBQLN2 and different amounts of Ub and Ub chains in $20 \mathrm{mM}$ NaPhosphate, $200 \mathrm{mM} \mathrm{NaCl}, 0.1 \mathrm{mM}$ TCEP, and $0.5 \mathrm{mM}$ EDTA (pH 6.8), spiked with UBQLN2 labelled with Dylight 650 and/or Ub chains labelled with Dylight 488. Samples were added to Eisco Labs Microscope Slides, with Single Concavity, and covered with MatTek coverslips that had been coated with $5 \%$ bovine serum albumin (BSA) to minimize changes due 
to surface interactions, and incubated coverslip-side down at $30^{\circ} \mathrm{C}$ for $10 \mathrm{~min}$. Phase separation was imaged on an ONI Nanoimager (Oxford Nanoimaging Ltd, Oxford, UK) equipped with a Hamamatsu SCMOS ORCA flash 4.0 V3 camera using an Olympus 100×/1.4 N.A. objective. Images were prepared using Fiji (Schindelin et al., 2012) and FigureJ plugin.

\section{Calculations of Dilute and Dense Phase Concentrations}

Samples were prepared on ice to contain $50 \mu \mathrm{L}$ of $70 \mu \mathrm{M}$ UBQLN2, $10 \mu \mathrm{M}$ of UBQLN2 labelled with Alexa Fluor 488, $5 \mu \mathrm{M}$ of Ub or Ub chains labelled with Dylight 650, and different amounts of Ub and Ub chains in $20 \mathrm{mM}$ NaPhosphate, $200 \mathrm{mM} \mathrm{NaCl}, 0.1 \mathrm{mM}$ TCEP, and $0.5 \mathrm{mM}$ EDTA (pH 6.8). Samples were incubated at $30^{\circ} \mathrm{C}$ for 10 minutes, then centrifuged at $10000 \times \mathrm{g}$ for 5 minutes at $30^{\circ} \mathrm{C}$. Without disrupting the pellet, as much of the supernatant as possible was transferred to a new tube. $8 \mu \mathrm{L}$ of $8 \mathrm{M}$ urea solution was added to the pellet. Tube containing pellet and urea was incubated for 1 hour at room temperature, vortexed and centrifuged. The volume of the mixture was determined by pipetting with a P10. The dense phase volume was determined by subtracting the total volume by $8 \mu \mathrm{L}$ of urea added. Absorbance at $493 \mathrm{~nm}$ (for Alexa Fluor 488/UBQLN2) and 655 nm (for Dylight 650/Ub chains) were recorded. Dense and dilute phase concentrations were determined with a standard curve for multiple concentrations of UBQLN2 mixed with UBQLN2 labelled with Alexa Fluor 488 and Ub chains mixed with Ub chains labelled with Dylight 650.

\section{NMR Spectroscopy}

Proteins were prepared in $20 \mathrm{mM}$ NaPhosphate buffer ( $\mathrm{pH} 6.8$ ), $0.5 \mathrm{mM}$ EDTA, $0.02 \% \mathrm{NaN}_{3}$, and $5 \% \mathrm{D}_{2} \mathrm{O} .{ }^{1} \mathrm{H}-{ }^{15} \mathrm{~N}$ SOFAST-HMQC was used for titration experiments of UBQLN2 constructs and polyUb, although additional ${ }^{1} \mathrm{H}-{ }^{15} \mathrm{~N}$ TROSY-HSQC experiments were collected at distinct Ub:UBQLN2 ratios. ${ }^{1} \mathrm{H}-{ }^{15} \mathrm{~N}$ TROSY-HSQC experiments were used for experiments involving fulllength UBQLN2. All NMR experiments were performed on a Bruker Avance III $800 \mathrm{MHz}$ spectrometer equipped with a TCl cryoprobe at $25^{\circ} \mathrm{C} .{ }^{1} \mathrm{H}-{ }^{15} \mathrm{~N}$ SOFAST-HMQC experiments were acquired using spectral widths of 16 and $30 \mathrm{ppm}$ in the direct ${ }^{1} \mathrm{H}$ and indirect ${ }^{15} \mathrm{~N}$ dimensions, using 2048 and 160 total points, respectively. NMRPipe (Delaglio et al., 1995) was used to process all NMR data, and peak analyses were performed using CCPNMR 2.5 (Vranken et al., 2005). Chemical shift perturbations (CSPs) were quantified as follows: $\Delta \delta=$ $\left[\left(\Delta \delta_{H}\right)^{2}+\left(\Delta \delta_{N} / 5\right)^{2}\right]^{1 / 2}$ where $\Delta \delta_{H}$ and $\Delta \delta_{N}$ are the differences in ${ }^{1} \mathrm{H}$ and ${ }^{15} \mathrm{~N}$ chemical shifts, respectively. 


\section{NMR Titration Experiments and $K_{d}$ Determination}

Unlabeled protein ligand (Ub4 in most cases) was titrated into $50 \mu \mathrm{M}$ samples of ${ }^{15} \mathrm{~N}$-labelled protein (UBQLN2), and the binding was monitored by recording ${ }^{1} \mathrm{H}-{ }^{15} \mathrm{~N}$ TROSY-HSQC or SOFAST-HMQC spectra as a function of ligand:protein ratios of concentrations. At any titration point, the observed CSP can be represented as a weighted average between the two values corresponding to the free $(\Delta \delta=0)$ and completely ligand-bound $\left(\Delta \delta=\Delta \delta_{\text {bound }}\right)$ states, so that $\Delta \delta$ $=\Delta \delta_{\text {bound }} \mathrm{p}_{\text {bound, }}$ where $\mathrm{p}_{\text {bound }}$ is the relative population of the bound state of the molecule. As only a single Ub can bind to a UBQLN2 molecule, we employed a single-site binding model to determine the binding affinity. Concentrations of Ub ligand were adjusted by the number of Ub units in the polyUb chain such that $50 \mu \mathrm{M}$ Ub4 was equal to $200 \mu \mathrm{M}$ Ub. After this correction, we used the single-site binding model:

$$
p_{\text {bound }}=\frac{\left(\left[P_{t}\right]+\left[L_{t}\right]+K_{d}\right)-\sqrt{\left(\left[P_{t}\right]+\left[L_{t}\right]+K_{d}\right)^{2}-4\left[P_{t}\right]\left[L_{t}\right]}}{2\left[P_{t}\right]}(\text { Equation 2) }
$$

where $\left[\mathrm{P}_{t}\right]$ and $\left[\mathrm{L}_{t}\right]$ are the UBQLN2 and Ub concentrations, respectively, and $\mathrm{K}_{d}$ is the dissociation equilibrium constant. Data fitting for each amide was performed using an in-house MATLAB program. Only residues with a CSP > $0.06 \mathrm{ppm}$ at the titration endpoint were considered for $K_{d}$ determination. Reported $K_{d}$ values were averages of residue-specific $\mathrm{K}_{d}$ values with errors reflecting standard deviation of these values.

\section{Sedimentation Velocity Analytical Ultracentrifugation}

Purified UBQLN2 487-624, K48-Ub4, K63-Ub4, or UBQLN2:Ub4 complexes were loaded into 3- or 12-mm two-sector charcoal-filled Epon centerpieces with sapphire windows. All experiments were carried out using a Beckman Coulter ProteomeLab XL-A analytical ultracentrifuge equipped with absorbance optics and a 4-hole An-60 Ti rotor at 60,000 rpm that was pre-equilibrated to $20^{\circ} \mathrm{C}$ prior to running the experiment. The samples were scanned with a zero second time interval between scans for 300 scans and analyzed by the continuous distribution $(c(s))$ method in the program SEDFIT (Schuck, 2000). Concentration profiles $(a(r, t))$ were modeled as the sum of Lamm Equation solutions scaled by a continuous distribution $c(s)$ as follows:

$$
\left.a(r, t) \cong \int_{S_{\min }}^{s_{\max }} c(s) \chi(s, D(s), r, t) d s, \quad \text { (Equation } 3\right)
$$

where $s$ is the sedimentation coefficient, $\chi(s, D(s), r, t)$ is the Lamm Equation solution that is dependent on $D(s)$, the corresponding diffusion coefficient, $r$, radius from the center of rotation, and $t$, the time from the beginning of the experiment (Padrick and Brautigam, 2011; Schuck, 2000). The program SEDNTERP (Laue et al., 1992) was used to calculate the buffer density 
$(1.0064 \mathrm{~mL} / \mathrm{g})$, viscosity $(0.009 \mathrm{P})$, and partial specific volume of UBQLN2 $(\bar{v}=0.7281)$, K48Ub4 (0.746), K63-Ub4 (0.746), K48-Ub4+UBQLN2 (0.7405), and K63-Ub4+UBQLN2 (0.7426), which were based on the amino acid sequences.

Multi-signal sedimentation velocity (MSSV) analysis was performed to determine the stoichiometry of the complex formed between UBQLN2 and Ub4. In MSSV, the standard $c(s)$ approach is modified to deconvolute the contributions of individual species with distinct spectral properties in a component distribution $c_{k}(s)$ where $k$ represents the individual components of a mixture. Here, the absorbance at wavelength $\lambda\left(a_{\lambda}(r, t)\right)$ is modeled as:

$$
\left.a_{\lambda}(r, t) \cong \sum_{k=1}^{K} \varepsilon_{\lambda}^{k} l \int_{S_{\min }}^{S_{\max }} c_{k}(s) \chi\left(s, D_{k}(s), r, t\right) d s \quad \text { (Equation } 4\right)
$$

where $l$ is the path-length, $K$ is the number of solutes present, and $c_{k}(s)$ is a continuous distribution for component $k$. The spectral properties of the individual proteins were first determined by SV-AUC using absorbance at $260 \mathrm{~nm}$ and $280 \mathrm{~nm}$. Global component $c_{k}(s)$ analysis of both signals was performed using the known $\varepsilon^{280}$ extinction coefficient as a reference to calibrate $\varepsilon^{260}$ for each protein (Table S4). The results indicate good spectral discrimination between UBQLN2 and both Ub4s with fitted spectral discrimination parameter $D_{\text {norm }}>0.236$ (Brautigam et al., 2013). MSSV of the UBQLN2:Ub4 mixtures were then collected at both 260 $\mathrm{nm}$ and $280 \mathrm{~nm}$ and globally fit using the multiwavelength discrete/continuous distribution analysis with mass constraints in SEDPHAT version 15-2b. Integration of the resulting $c_{k}(s)$ distributions revealed the molar signal increment for each protein under a peak at a given sedimentation value (Figure S4). All plots were created with GUSSI (Brautigam, 2015).

To determine the statistical significance of the resulting molar ratios, global $c_{k}(s)$ analyses were conducted by constraining the molar ratio to different values and determining if the resulting fit to the data $\chi_{t}^{2}$ is significantly worse than the unconstrained analysis $\chi^{2}$, using the built in F-statistics calculator to determine the critical $\chi^{2}$ at $1 \sigma$ and $2 \sigma$. Fixed molar ratios with $\chi_{t}^{2}$ values exceeding the critical $\chi^{2}$ value at $2 \sigma$ (dashed red line in Figure S4) were deemed significantly different from that of the unconstrained analysis and rejected (Padrick and Brautigam, 2011).

\section{SEC-MALS-SAXS Experiments:}

SAXS was performed at BioCAT (beamline 18ID at the Advanced Photon Source, Chicago) with in-line size exclusion chromatography (SEC) to separate sample from aggregates and other contaminants thus ensuring optimal sample quality and multiangle light scattering (MALS), dynamic light scattering (DLS) and refractive index measurement (RI)) for additional biophysical 
characterization (SEC-MALS-SAXS). All protein samples were prepared in $20 \mathrm{mM}$ NaPhosphate buffer at pH 6.8 containing 0.5 mM EDTA and $0.02 \% \mathrm{NaN}_{3}$ (See Table S4 for details). The samples were loaded on a Superdex 200 10/300 Increase column (Cytiva) at a temperature of $20^{\circ} \mathrm{C}$ run by a 1260 Infinity II HPLC (Agilent Technologies) at $0.6 \mathrm{ml} / \mathrm{min}$. The flow passed through (in order) the Agilent UV detector, a MALS detector and a DLS detector (DAWN Helios II, Wyatt Technologies), and an RI detector (Optilab T-rEX, Wyatt). The flow then went through the SAXS flow cell. The flow cell consists of a $1.0 \mathrm{~mm}$ ID quartz capillary with 20 $\mu \mathrm{m}$ walls. A coflowing buffer sheath is used to separate sample from the capillary walls, helping prevent radiation damage (Kirby et al., 2016). Scattering intensity was recorded using a Pilatus3 $\mathrm{X} 1 \mathrm{M}$ (Dectris) detector which was placed $3.6 \mathrm{~m}$ from the sample giving us access to a q-range of $0.003 \AA^{-1}$ to $0.35 \AA^{-1}$. $0.5 \mathrm{~s}$ exposures were acquired every $2 \mathrm{~s}$ during elution and data was reduced using BioXTAS RAW 2.1.1 (Hopkins et al., 2017). Buffer blanks were created by averaging regions flanking the elution peak (see Figure S5) and subtracted from exposures selected from the elution peak to create the $\mathrm{I}(q)$ vs $q$ curves used for subsequent analyses. Molecular weights and hydrodynamic radii were calculated from the MALS and DLS data, respectively, using the ASTRA 7 software (Wyatt). Additionally, $R_{g}$ and $I(0)$ values were obtained using the entire q-range of the data by calculating the distance distribution functions, $P(r)$ versus $r$, using GNOM (Svergun, 1992). 


\section{References}

Akutsu, M., Dikic, I., and Bremm, A. (2016). Ubiquitin chain diversity at a glance. J. Cell Sci. jes. 183954.

Alexander, E.J., Niaki, A.G., Zhang, T., Sarkar, J., Liu, Y., Nirujogi, R.S., Pandey, A., Myong, S., and Wang, J. (2018). Ubiquilin 2 modulates ALS/FTD-linked FUS-RNA complex dynamics and stress granule formation. Proc. Natl. Acad. Sci. 115, E11485-E11494.

Alshareedah, I., Kaur, T., Ngo, J., Seppala, H., Kounatse, L.-A.D., Wang, W., Moosa, M.M., and Banerjee, P.R. (2019). Interplay between Short-Range Attraction and Long-Range Repulsion Controls Reentrant Liquid Condensation of Ribonucleoprotein-RNA Complexes. J. Am. Chem. Soc. $141,14593-14602$.

Banerjee, P.R., Milin, A.N., Moosa, M.M., Onuchic, P.L., and Deniz, A.A. (2017). Reentrant Phase Transition Drives Dynamic Substructure Formation in Ribonucleoprotein Droplets. Angew. Chem. Int. Ed. 56, 11354-11359.

Beal, R., Deveraux, Q., Xia, G., Rechsteiner, M., and Pickart, C. (1996). Surface hydrophobic residues of multiubiquitin chains essential for proteolytic targeting. Proc. Natl. Acad. Sci. U. S. A. $93,861-866$.

Boughton, A.J., Krueger, S., and Fushman, D. (2019). Branching via K11 and K48 Bestows Ubiquitin Chains with a Unique Interdomain Interface and Enhanced Affinity for Proteasomal Subunit Rpn1. Structure 28, 29-43.E6.

Brautigam, C.A. (2015). Chapter Five - Calculations and Publication-Quality Illustrations for Analytical Ultracentrifugation Data. In Methods in Enzymology, J.L. Cole, ed. (Academic Press), pp. 109-133.

Brautigam, C.A., Padrick, S.B., and Schuck, P. (2013). Multi-Signal Sedimentation Velocity Analysis with Mass Conservation for Determining the Stoichiometry of Protein Complexes. PLOS ONE 8, e62694.

Bremm, A., Freund, S.M.V., and Komander, D. (2010). Lys11-linked ubiquitin chains adopt compact conformations and are preferentially hydrolyzed by the deubiquitinase Cezanne. Nat. Struct. Mol. Biol. 17, 939-947.

Buchan, J.R., Kolaitis, R.-M., Taylor, J.P., and Parker, R. (2013). Eukaryotic Stress Granules Are Cleared by Autophagy and Cdc48/VCP Function. Cell 153, 1461-1474.

Cabe, M., Rademacher, D.J., Karlsson, A.B., Cherukuri, S., and Bakowska, J.C. (2018). PB1 and UBA domains of p62 are essential for aggresome-like induced structure formation. Biochem. Biophys. Res. Commun. 503, 2306-2311.

Carvalho, A.F., Pinto, M.P., Grou, C.P., Vitorino, R., Domingues, P., Yamao, F., Sá-Miranda, C., and Azevedo, J.E. (2011). High-Yield Expression in Escherichia coli and Purification of Mouse Ubiquitin-Activating Enzyme E1. Mol. Biotechnol. 51, 254-261. 
Castañeda, C.A., Chaturvedi, A., Camara, C.M., Curtis, J.E., Krueger, S., and Fushman, D. (2016a). Linkage-specific conformational ensembles of non-canonical polyubiquitin chains. Phys. Chem. Chem. Phys. PCCP 18, 5771-5788.

Castañeda, C.A., Dixon, E.K., Walker, O., Chaturvedi, A., Nakasone, M.A., Curtis, J.E., Reed, M.R., Krueger, S., Cropp, T.A., and Fushman, D. (2016b). Linkage via K27 Bestows Ubiquitin Chains with Unique Properties among Polyubiquitins. Structure 24, 423-436.

Ceballos-Diaz, C., Rosario, A.M., Park, H.-J., Chakrabarty, P., Sacino, A., Cruz, P.E., Siemienski, Z., Lara, N., Moran, C., Ravelo, N., et al. (2015). Viral expression of ALS-linked ubiquilin-2 mutants causes inclusion pathology and behavioral deficits in mice. Mol. Neurodegener. 10, 25.

Chen, T., Huang, B., Shi, X., Gao, L., and Huang, C. (2018). Mutant UBQLN2P497H in motor neurons leads to ALS-like phenotypes and defective autophagy in rats. Acta Neuropathol. Commun. 6, 122.

Choi, J.-M., Dar, F., and Pappu, R.V. (2019). LASSI: A lattice model for simulating phase transitions of multivalent proteins. PLOS Comput. Biol. 15, e1007028.

Dao, T.P., and Castañeda, C.A. (2020). Ubiquitin-Modulated Phase Separation of Shuttle Proteins: Does Condensate Formation Promote Protein Degradation? BioEssays 42, 2000036.

Dao, T.P., Kolaitis, R.-M., Kim, H.J., O’Donovan, K., Martyniak, B., Colicino, E., Hehnly, H., Taylor, J.P., and Castañeda, C.A. (2018). Ubiquitin Modulates Liquid-Liquid Phase Separation of UBQLN2 via Disruption of Multivalent Interactions. Mol. Cell 69, 965-978.e6.

Dao, T.P., Martyniak, B., Canning, A.J., Lei, Y., Colicino, E.G., Cosgrove, M.S., Hehnly, H., and Castañeda, C.A. (2019). ALS-Linked Mutations Affect UBQLN2 Oligomerization and Phase Separation in a Position- and Amino Acid-Dependent Manner. DTR 27, 937-951.e5.

Delaglio, F., Grzesiek, S., Vuister, G.W., Zhu, G., Pfeifer, J., and Bax, A. (1995). NMRPIPE - A multidimensional spectral processing system based on UNIX pipes. J Biomol NMR 6, 277-293.

Deng, H.-X., Chen, W., Hong, S.-T., Boycott, K.M., Gorrie, G.H., Siddique, N., Yang, Y., Fecto, F., Shi, Y., Zhai, H., et al. (2011). Mutations in UBQLN2 cause dominant X-linked juvenile and adult onset ALS and ALS/dementia. Nature 477, 211-215.

Dikic, I. (2017). Proteasomal and Autophagic Degradation Systems. Annu. Rev. Biochem. 86, 193-224.

Eddins, M.J., Varadan, R., Fushman, D., Pickart, C.M., and Wolberger, C. (2007). Crystal Structure and Solution NMR Studies of Lys48-linked Tetraubiquitin at Neutral pH. J. Mol. Biol. $367,204-211$.

Fecto, F., Gorrie, G., Zhai, H., Liu, E., Deng, H.-X., and Siddique, T. (2013). Impaired Activity of the Ubiquitin-Proteasome System in Transgenic Mice Expressing ALS/Dementia-Linked Mutant UBQLN2 (P02.170). Neurology 80, P02.170. 
Franke, D., Jeffries, C.M., and Svergun, D.I. (2015). Correlation Map, a goodness-of-fit test for one-dimensional X-ray scattering spectra. Nat. Methods 12, 419-422.

Fu, A., Cohen-Kaplan, V., Avni, N., Livneh, I., and Ciechanover, A. (2021). p62-containing, proteolytically active nuclear condensates, increase the efficiency of the ubiquitin-proteasome system. Proc. Natl. Acad. Sci. 118.

Gerson, J.E., Linton, H., Xing, J., Sutter, A.B., Kakos, F.S., Ryou, J., Liggans, N., Sharkey, L.M., Safren, N., Paulson, H.L., et al. (2021). Shared and divergent phase separation and aggregation properties of brain-expressed ubiquilins. Sci. Rep. 11, 287.

Guillén-Boixet, J., Kopach, A., Holehouse, A.S., Wittmann, S., Jahnel, M., Schlüßler, R., Kim, K., Trussina, I.R.E.A., Wang, J., Mateju, D., et al. (2020). RNA-Induced Conformational Switching and Clustering of G3BP Drive Stress Granule Assembly by Condensation. Cell 181, 346-361.e17.

Gwon, Y., Maxwell, B.A., Kolaitis, R.-M., Zhang, P., Kim, H.J., and Taylor, J.P. (2021). Ubiquitination of G3BP1 mediates stress granule disassembly in a context-specific manner. Science 372.

Haglund, K., and Dikic, I. (2012). The Role of Ubiquitylation in Receptor Endocytosis and Endosomal Sorting. J. Cell Sci. 125, 265-275.

Halloran, M., Ragagnin, A.M.G., Vidal, M., Parakh, S., Yang, S., Heng, B., Grima, N., Shahheydari, H., Soo, K.-Y., Blair, I., et al. (2020). Amyotrophic lateral sclerosis-linked UBQLN2 mutants inhibit endoplasmic reticulum to Golgi transport, leading to Golgi fragmentation and ER stress. Cell. Mol. Life Sci. 77, 3859-3873.

Harman, C.A., and Monteiro, M.J. (2019). The specificity of ubiquitin binding to ubiquilin-1 is regulated by sequences besides its UBA domain. Biochim. Biophys. Acta BBA - Gen. Subj.

Hipp, M.S., Park, S.-H., and Hartl, F.U. (2014). Proteostasis impairment in protein-misfolding and -aggregation diseases. Trends Cell Biol. 24, 506-514.

Hjerpe, R., Bett, J.S., Keuss, M.J., Solovyova, A., McWilliams, T.G., Johnson, C., Sahu, I., Varghese, J., Wood, N., Wightman, M., et al. (2016). UBQLN2 Mediates AutophagyIndependent Protein Aggregate Clearance by the Proteasome. Cell 166, 935-949.

Hoeller, D., Hecker, C.-M., Wagner, S., Rogov, V., Dötsch, V., and Dikic, I. (2007). E3Independent Monoubiquitination of Ubiquitin-Binding Proteins. Mol. Cell 26, 891-898.

Hopkins, J.B., Gillilan, R.E., and Skou, S. (2017). BioXTAS RAW: improvements to a free open-source program for small-angle X-ray scattering data reduction and analysis. J. Appl. Crystallogr. 50, 1545-1553.

Kaiser, S.E., Riley, B.E., Shaler, T.A., Trevino, R.S., Becker, C.H., Schulman, H., and Kopito, R.R. (2011). Protein standard absolute quantification (PSAQ) method for the measurement of cellular ubiquitin pools. Nat. Methods $8,691-696$. 
Kirby, N., Cowieson, N., Hawley, A.M., Mudie, S.T., McGillivray, D.J., Kusel, M., SamardzicBoban, V., and Ryan, T.M. (2016). Improved radiation dose efficiency in solution SAXS using a sheath flow sample environment. Acta Crystallogr. Sect. Struct. Biol. 72, 1254-1266.

Komander, D., and Rape, M. (2012). The Ubiquitin Code. Annu. Rev. Biochem. 81, 203-229.

Labbadia, J., and Morimoto, R.I. (2015). The biology of proteostasis in aging and disease. Annu. Rev. Biochem. 84, 435-464.

Laue, T.M., Shah, B.D., Ridgeway, T.M., and Pelletier, S.L. (1992). Computer-aided interpretation of analytical sedimentation data for proteins. In Analytical Ultracentrifugation in Biochemistry and Polymer Science, (Cambridge, UK: The Royal Society of Chemistry), pp. 90125.

Levin-Kravets, O., Shohat, N., and Prag, G. (2015). Tetrameric Assembly of Monoubiquitin Accurately Mimics the Lys11 Polyubiquitin Chain Structure. Biochemistry 54, 4704-4710.

Lin, B.C., Higgins, N.R., Phung, T.H., and Monteiro, M.J. UBQLN proteins in health and disease with a focus on UBQLN2 in ALS/FTD. FEBS J. $n / a$.

Linares, J.F., Duran, A., Yajima, T., Pasparakis, M., Moscat, J., and Diaz-Meco, M.T. (2013). K63 Polyubiquitination and Activation of mTOR by the p62-TRAF6 Complex in NutrientActivated Cells. Mol. Cell 51, 283-296.

Long, J., Gallagher, T.R.A., Cavey, J.R., Sheppard, P.W., Ralston, S.H., Layfield, R., and Searle, M.S. (2008). Ubiquitin Recognition by the Ubiquitin-associated Domain of p62 Involves a Novel Conformational Switch. J. Biol. Chem. 283, 5427-5440.

Lowe, J., Blanchard, A., Morrell, K., Lennox, G., Reynolds, L., Billett, M., Landon, M., and Mayer, R.J. (1988). Ubiquitin is a common factor in intermediate filament inclusion bodies of diverse type in man, including those of Parkinson's disease, Pick's disease, and Alzheimer's disease, as well as Rosenthal fibres in cerebellar astrocytomas, cytoplasmic bodies in muscle, and mallory bodies in alcoholic liver disease. J. Pathol. 155, 9-15.

Lu, K., den Brave, F., and Jentsch, S. (2017). Receptor oligomerization guides pathway choice between proteasomal and autophagic degradation. Nat. Cell Biol. 19, 732-739.

Manetto, V., Perry, G., Tabaton, M., Mulvihill, P., Fried, V.A., Smith, H.T., Gambetti, P., and Autilio-Gambetti, L. (1988). Ubiquitin is associated with abnormal cytoplasmic filaments characteristic of neurodegenerative diseases. Proc. Natl. Acad. Sci. 85, 4501-4505.

Molliex, A., Temirov, J., Lee, J., Coughlin, M., Kanagaraj, A.P., Kim, H.J., Mittag, T., and Taylor, J.P. (2015). Phase Separation by Low Complexity Domains Promotes Stress Granule Assembly and Drives Pathological Fibrillization. Cell 163, 123-133.

Morimoto, D., Walinda, E., Fukada, H., Sou, Y.-S., Kageyama, S., Hoshino, M., Fujii, T., Tsuchiya, H., Saeki, Y., Arita, K., et al. (2015). The unexpected role of polyubiquitin chains in the formation of fibrillar aggregates. Nat. Commun. 6, 6116. 
Nathan, J.A., Tae Kim, H., Ting, L., Gygi, S.P., and Goldberg, A.L. (2013). Why do cellular proteins linked to K63-polyubiquitin chains not associate with proteasomes? EMBO J. 32, 552565.

Nedelsky, N.B., and Taylor, J.P. (2019). Bridging biophysics and neurology: aberrant phase transitions in neurodegenerative disease. Nat. Rev. Neurol. 1.

Olzmann, J.A., Li, L., Chudaev, M.V., Chen, J., Perez, F.A., Palmiter, R.D., and Chin, L.-S. (2007). Parkin-mediated K63-linked polyubiquitination targets misfolded DJ-1 to aggresomes via binding to HDAC6. J. Cell Biol. 178, 1025-1038.

Padrick, S.B., and Brautigam, C.A. (2011). Evaluating the stoichiometry of macromolecular complexes using multisignal sedimentation velocity. Methods San Diego Calif 54, 39-55.

Park, C.-W., and Ryu, K.-Y. (2014). Cellular ubiquitin pool dynamics and homeostasis. BMB Rep. 47, 475-482.

Patel, A., Lee, H.O., Jawerth, L., Maharana, S., Jahnel, M., Hein, M.Y., Stoynov, S., Mahamid, J., Saha, S., Franzmann, T.M., et al. (2015). A Liquid-to-Solid Phase Transition of the ALS Protein FUS Accelerated by Disease Mutation. Cell 162, 1066-1077.

Piiadov, V., Ares de Araújo, E., Oliveira Neto, M., Craievich, A.F., and Polikarpov, I. (2019). SAXSMoW 2.0: Online calculator of the molecular weight of proteins in dilute solution from experimental SAXS data measured on a relative scale. Protein Sci. Publ. Protein Soc. 28, 454463.

Piper, R.C., Dikic, I., and Lukacs, G.L. (2014). Ubiquitin-Dependent Sorting in Endocytosis. Cold Spring Harb. Perspect. Biol. 6, a016808.

Raasi, S., Varadan, R., Fushman, D., and Pickart, C.M. (2005). Diverse polyubiquitin interaction properties of ubiquitin-associated domains. Nat. Struct. Mol. Biol. 12, 708-714.

Rambo, R.P., and Tainer, J.A. (2013). Accurate assessment of mass, models and resolution by small-angle scattering. Nature 496, 477-481.

Reber, J.M., and Mangerich, A. (2021). Why structure and chain length matter: on the biological significance underlying the structural heterogeneity of poly(ADP-ribose). Nucleic Acids Res. 49, 8432-8448.

Riley, B.E., Kaiser, S.E., Shaler, T.A., Ng, A.C.Y., Hara, T., Hipp, M.S., Lage, K., Xavier, R.J., Ryu, K.-Y., Taguchi, K., et al. (2010). Ubiquitin accumulation in autophagy-deficient mice is dependent on the Nrf2-mediated stress response pathway: a potential role for protein aggregation in autophagic substrate selection. J. Cell Biol. 191, 537-552.

Ruff, K.M., Dar, F., and Pappu, R.V. (2021a). Ligand effects on phase separation of multivalent macromolecules. Proc. Natl. Acad. Sci. 118. 
Ruff, K.M., Dar, F., and Pappu, R.V. (2021b). Polyphasic linkage and the impact of ligand binding on the regulation of biomolecular condensates. Biophys. Rev. 2, 021302.

Ryan, V.H., and Fawzi, N.L. (2019). Physiological, pathological, and targetable membraneless organelles in neurons. Trends Neurosci. 42, 693-708.

Sanders, D.W., Kedersha, N., Lee, D.S.W., Strom, A.R., Drake, V., Riback, J.A., Bracha, D., Eeftens, J.M., Iwanicki, A., Wang, A., et al. (2020). Competing Protein-RNA Interaction Networks Control Multiphase Intracellular Organization. Cell 181, 306-324.e28.

Schindelin, J., Arganda-Carreras, I., Frise, E., Kaynig, V., Longair, M., Pietzsch, T., Preibisch, S., Rueden, C., Saalfeld, S., Schmid, B., et al. (2012). Fiji: an open-source platform for biological-image analysis. Nat. Methods 9, 676-682.

Schuck, P. (2000). Size-distribution analysis of macromolecules by sedimentation velocity ultracentrifugation and lamm equation modeling. Biophys. J. 78, 1606-1619.

Sharkey, L.M., Sandoval-Pistorius, S.S., Moore, S.J., Gerson, J.E., Komlo, R., Fischer, S., Negron-Rios, K.Y., Crowley, E.V., Padron, F., Patel, R., et al. (2020). Modeling UBQLN2mediated neurodegenerative disease in mice: Shared and divergent properties of wild type and mutant UBQLN2 in phase separation, subcellular localization, altered proteostasis pathways, and selective cytotoxicity. Neurobiol. Dis. 143, 105016.

Sontag, E.M., Samant, R.S., and Frydman, J. (2017). Mechanisms and Functions of Spatial Protein Quality Control. Annu. Rev. Biochem. 86, 97-122.

Sun, D., Wu, R., Zheng, J., Li, P., and Yu, L. (2018). Polyubiquitin chain-induced p62 phase separation drives autophagic cargo segregation. Cell Res. 28, 405-415.

Svergun, D.I. (1992). Determination of the regularization parameter in indirect-transform methods using perceptual criteria. J. Appl. Crystallogr. 25, 495-503.

Tenno, T., Fujiwara, K., Tochio, H., Iwai, K., Morita, E.H., Hayashi, H., Murata, S., Hiroaki, H., Sato, M., Tanaka, K., et al. (2004). Structural basis for distinct roles of Lys63- and Lys48-linked polyubiquitin chains. Genes Cells 9, 865-875.

Turco, E., Witt, M., Abert, C., Bock-Bierbaum, T., Su, M.-Y., Trapannone, R., Sztacho, M., Danieli, A., Shi, X., Zaffagnini, G., et al. (2019). FIP200 Claw Domain Binding to p62 Promotes Autophagosome Formation at Ubiquitin Condensates. Mol. Cell 74, 330-346.e11.

Vendruscolo, M. (2012). Proteome folding and aggregation. Curr. Opin. Struct. Biol. 22, 138143.

Vranken, W.F., Boucher, W., Stevens, T.J., Fogh, R.H., Pajon, A., Llinas, M., Ulrich, E.L., Markley, J.L., Ionides, J., and Laue, E.D. (2005). The CCPN data model for NMR spectroscopy: Development of a software pipeline. Proteins Struct. Funct. Bioinforma. 59, 687-696. 
Williamson, M.P. (2013). Using chemical shift perturbation to characterise ligand binding. Prog. Nucl. Magn. Reson. Spectrosc. 73, 1-16.

Wyman, J., and Gill, S.J. (1980). Ligand-linked phase changes in a biological system: applications to sickle cell hemoglobin. Proc. Natl. Acad. Sci. 77, 5239-5242.

Yang, P., Mathieu, C., Kolaitis, R.-M., Zhang, P., Messing, J., Yurtsever, U., Yang, Z., Wu, J., Li, Y., Pan, Q., et al. (2020). G3BP1 Is a Tunable Switch that Triggers Phase Separation to Assemble Stress Granules. Cell 181, 325-345.e28.

Yang, Y., Jones, H.B., Dao, T.P., and Castañeda, C.A. (2019). Single Amino Acid Substitutions in Stickers, but Not Spacers, Substantially Alter UBQLN2 Phase Transitions and Dense Phase Material Properties. J. Phys. Chem. B 123, 3618-3629.

Yasuda, S., Tsuchiya, H., Kaiho, A., Guo, Q., Ikeuchi, K., Endo, A., Arai, N., Ohtake, F., Murata, S., Inada, T., et al. (2020). Stress- and ubiquitylation-dependent phase separation of the proteasome. Nature 578, 296-300.

Ye, Y., Blaser, G., Horrocks, M.H., Ruedas-Rama, M.J., Ibrahim, S., Zhukov, A.A., Orte, A., Klenerman, D., Jackson, S.E., and Komander, D. (2012). Ubiquitin chain conformation regulates recognition and activity of interacting proteins. Nature 492, 266-270.

Zaffagnini, G., Savova, A., Danieli, A., Romanov, J., Tremel, S., Ebner, M., Peterbauer, T., Sztacho, M., Trapannone, R., Tarafder, A.K., et al. (2018). p62 filaments capture and present ubiquitinated cargos for autophagy. EMBO J. 37, e98308.

Zhang, D., Raasi, S., and Fushman, D. (2008). Affinity Makes the Difference: Nonselective Interaction of the UBA Domain of Ubiquilin-1 with Monomeric Ubiquitin and Polyubiquitin Chains. J. Mol. Biol. 377, 162-180.

Zhang, Q., Huang, H., Zhang, L., Wu, R., Chung, C.-I., Zhang, S.-Q., Torra, J., Schepis, A., Coughlin, S.R., Kornberg, T.B., et al. (2018). Visualizing Dynamics of Cell Signaling In Vivo with a Phase Separation-Based Kinase Reporter. Mol. Cell 69, 334-346.e4.

Zheng, T., Yang, Y., and Castañeda, C.A. (2020). Structure, dynamics and functions of UBQLNs: at the crossroads of protein quality control machinery. Biochem. J. 477, 3471-3497.

Zientara-Rytter, K., and Subramani, S. (2019). The Roles of Ubiquitin-Binding Protein Shuttles in the Degradative Fate of Ubiquitinated Proteins in the Ubiquitin-Proteasome System and Autophagy. Cells 8 . 